Anastasiia Samoilikova,

Ph.D., Sumy State University, Ukraine

ORCID ID, 0000-0001-8639-5282

email: av.samoilikova@finance.sumdu.edu.ua

Serhiy Lieonov,

D.Sc., Professor, Sumy State University, Ukraine

(iD) ORCID ID, 0000-0001-5639-3008

email: s.lieonov@uabs.sumdu.edu.ua

Alida Huseynova,

Ph.D., Azerbaijan State University of Economics, Republic of Azerbaijan

iD ORCID ID, 0000-0001-8110-3025

email: alida_gusenova@unec.edu.az

Correspondence author: av.samoilikova@finance.sumdu.edu.ua

\title{
TAX INCENTIVES FOR INNOVATION IN THE CONTEXT OF MACROECONOMIC STABILITY: AN ANALYSIS OF CAUSALITY
}

Abstract. The article deals with the topical issue of $R \& D$ tax incentives and their impact on the level of innovation development and macroeconomic stability. The research is based on causality analysis and estimation of the strength, time lags and directions of mutual influence of $R \& D$ tax incentives and macro indicators. Systematization literary sources and approaches for solving this problem indicates that $R \& D$ tax incentives are studied in fragments in the context of macroeconomic stability. The research's main purpose is to improve the methodological bases of substantiation of the choice of relevant instruments of innovation stimulation considering causal relations of $R \& D$ tax incentives and macro indicators. The paper presents the results of dynamic analysis of $R \& D$ tax incentives in 13 European countries, for which OECD statistics for 2007-2017 are freely available. The significance, strength, and nature of the relationship between these indicators and the following macro indicators are determined: the level of the country's innovation development, the share of investment in GDP (in general and in the corporate sector in particular), net international investment position, the share of the business sector in the cost structure of R\&D. Pearson and Spearman correlation coefficients were calculated depending on the variable subordination to the law of normal distribution (verified by the Shapiro - Wilk test) on the admissible calculation interval taking into account time lags from 0 years to 3 years. The causality of the studied indicators was established using the Granger causality test. The calculations are important for the prioritization of instruments for the implementation of innovation support. The highest priority should be given to the establishment of tax incentives for $R \& D$, as this tool's impact on all studied macro indicators in most countries was direct. Its effect was manifested in the shortest possible time (with a lag of 0-3 years). The second priority should be given to setting hidden rates of business tax subsidies on R\&D, as this indicator's impact on most of the studied indicators was statistically significant and direct with a time lag of 0-3 years. The paper substantiates the inefficiency of direct public financial support, as the impact of this indicator on most of the analyzed macro-indicators was reversed with a lag of 0-2 years. Thus, it is more expedient for the state to help entrepreneurs by providing tax benefits to provide innovation development and macro stability than through direct reimbursement of costs. Moreover, lag regression models were built for those countries where identified links were the most important (Belgium, Denmark, the Netherlands, and the Czech Republic). They take into account inflation rates and interest rates on long-term liabilities and the number of labour resources in the country as control variables.

Keywords: causal relations, Granger causality test, innovation, investment position, macro indicators, macroeconomic stability, R\&D tax expenditure, R\&D, tax incentives, tax subsidies.

Cite as: Samoilikova, A., Lieonov, S., \& Huseynova, A. (2021). Tax Incentives for Innovation in the Context of Macroeconomic Stability: an Analysis of Causality. Marketing and Management of Innovations, 1, 135157. http://doi.org/10.21272/mmi.2021.1-11 

Analysis of Causality

Introduction. The importance of macroeconomic stability in any country is beyond doubt. However, in the context of global challenges of an economic nature and, for example, under the influence of the coronavirus pandemic, indicators of macroeconomic stability in Ukraine and Azerbaijan show lagging behind targets compared to developed European countries. Under such conditions, we consider tax incentives for innovation to be relevant in increasing macroeconomic stability.

Indicators of tax and fiscal stimulus are an important component of fiscal policy to ensure the innovative development of the world, as research and development (R\&D) provide both private and social effect (and many times greater than private) (Jones and Williams, 1998; Czyżewski et al., 2019; Miśkiewicz \& Wolniak, 2020; Baranovskyi, 2020), which leads to government intervention in the field of innovation. Besides, tax incentives are tools to stimulate R\&D in the private sector directly.

As tax and budget-investment incentives for innovation have become widespread in developed EU countries but are currently underdeveloped in Ukraine, Azerbaijan, and many other post-Soviet countries, this issue requires further fundamental and applied research.

The purpose of the article is to improve the methodological basis for substantiating the choice of relevant instruments to stimulate innovation development, considering the areas of mutual influence of tax and budget-investment stimulation of innovation development on many indicators of macroeconomic growth.

Literature Review. Several scholars have studied some aspects of R\&D tax incentives. Bloom et al. (2002) examined the impact of fiscal stimulus on R\&D investment by estimating the econometric model of R\&D investment based on tax changes and R\&D expenditures in nine OECD countries over 19 years (1979-1997) and conclude that R\&D tax incentives would increase their intensity. Scientists empirically confirm that a $10 \%$ drop in R\&D costs stimulates a little more than 1\% growth in R\&D in the short term and a little less than 10\% growth in R\&D in the long run. Assessing the effectiveness of fiscal stimulus R\&D is also covered in the work of Hall and Reenen (2000), Lee (2011), Hrytsenko et al (2018), Kuznetsova and Pohorelenko (2020), Tkachenko et al. (2019) and others.

Fazio et al. (2020) substantiated the impact of state tax credits for R\&D on quantitative and qualitative entrepreneurship indicators, pointing to the potential of state tax crediting of innovations to stimulate entrepreneurship. Klemm (2009) analyzes various tax incentives for business investment, positioning tax benefits in response to tax competition - the main driver of tax reforms. It provides theoretical and empirical conclusions on the pros and cons of tax benefits and more. Tassey (1996) compares tax incentives for R\&D with direct public funding, justifying public policy options in the field of R\&D.

Corchuelo and Martínez-Ros (2010) characterized the benefits that firms receive from fiscal R\&D incentives and the overall impact of this type of tool on innovation performance. Emphasizing that tax incentives are not widespread and rarely used by firms, researchers found that large innovative firms are more likely to use tax incentives (and significantly affect this). In contrast, small and medium-sized ones face particular barriers to their use.

Hodzic and Becic (2016) examined the tax incentives for R\&D of large Croatian companies. They emphasized the need to review legislation to remove obstacles created by bureaucratic barriers and over-regulation in the process of tax incentives for R\&D (primarily as a means of reducing the income tax base). Further, encourage the production of new goods and services, create new jobs, and stimulate economic growth. Cappelen et al. (2010) investigated the Norwegian R\&D tax credit scheme, which encourages a large number of firms to invest more in R\&D and has a positive effect, including for small firms with little research and development experience. FurKoga (2003) drew attention to tax incentives' peculiarities for large and small, and medium-sized enterprises.

$\mathrm{Li}$ and Du (2016) view tax incentives for firm research as an important tool for governments seeking to address the «market failure» of R\&D investment. The authors use China's example to study the 

Analysis of Causality

impact of adjusting costs on the incentive effects of R\&D tax policy, arguing that R\&D tax incentives have a positive impact on the level of investment in innovation. Still, the effect gradually decreases with increasing adjustment costs. Guceri (2016) assessed the impact of R\&D tax policy based on the experience of R\&D tax incentives for innovative firms in the US, UK, France, China and Korea (Guceri, 2016, Guceri and Liu, 2015).

Tiutiunyk et al. (2019) investigate innovations in the management of tax gaps in the economy and estimate the volume of tax gaps in the economy in the country's foreign economic activity as one of the tools for minimizing tax liabilities.

Particular attention was paid to developing countries: what incentives could be used in them; which makes them useful; how much they should cost (James, 2013).

However, despite the available scientific achievements, tax incentives in the field of R\&D were studied in fragments. Therefore, it requires consideration of the latest trends and the solution of existing problems, which caused the urgency of further research.

Methodology and research methods. This study applied the methods of correlation and regression analysis using the STATA software package for the sample from 13 European countries for 2007-2017 (limited calculations in 2017 due to the availability of information on open information portals of The Organization for Economic Co-operation and Development, the World Bank, EU Statistical Office) to confirm the hypothesis on the impact of the tax and budget-investment instruments of the innovation stimulation on the innovation and investment development of the country.

The nature of the distribution of the studied indicators was assessed using the Shapiro-Wilk test (Shapiro and Wilk, 1965). These results allowed choosing the calculating method of the correlation coefficient: Pearson - for indicators subject to the law of normal distribution (Pearson, 1896), or Spearman - for indicators that do not obey normal distribution (Spearman, 1987). The strength and nature of the relationship between the indices were established through a correlation analysis. It revealed the duration of time lags, after which this relationship is the most statistically significant. Granger causality test (Granger, 1969, Lopez and Weber, 2017, Tastan, 2015) was used to assess the relationship between tax and budget-investment instruments of the innovation stimulation and macro indicators of innovation investment development.

There are lag models of linear regression (Strielkowski \& Höschle, 2016; Simionescu et al., 2017; Vovchak et al., 2018; Savchenko et al., 2019) to determine the impact of the tax and budget-investment instruments on innovation and investment development in certain countries, where it was the most significant (Belgium, Denmark, the Netherlands, and the Czech Republic).

Results. To conduct this study, a sample of 13 European countries was formed, for which OECD statistics for 2007-2017 are available in free access for all studied indicators of tax incentives.

Actual tax subsidy rates on R\&D expenditures (Table 1) are one of the indicators of tax incentives calculated by the Organization for Economic Co-operation and Development (OECD) for OECD member countries and individual non-OECD countries (Warda, 2001; OECD Science, Technology and R\&D Statistics, OECD R\&D Tax Incentive Database).

Table 1. Implied tax subsidy rates on R\&D expenditures, $\%$

\begin{tabular}{|c|c|c|c|c|c|c|c|c|c|c|c|c|}
\hline Country & The firm's size & 2007 & 2008 & 2009 & 2010 & 2011 & 2012 & 2013 & 2014 & 2015 & 2016 & 2017 \\
\hline \multirow[b]{2}{*}{ Belgium } & SME & 0,10 & 0,13 & 0,15 & 0,15 & 0,15 & 0,15 & 0,16 & 0,16 & 0,16 & 0,16 & 0,16 \\
\hline & LF & 0,10 & 0,13 & 0,15 & 0,15 & 0,15 & 0,15 & 0,16 & 0,16 & 0,16 & 0,16 & 0,16 \\
\hline The Czech & SME & 0,27 & 0,23 & 0,21 & 0,20 & 0,20 & 0,20 & 0,20 & 0,21 & 0,21 & 0,21 & 0,21 \\
\hline Republic & LF & 0,27 & 0,23 & 0,21 & 0,20 & 0,20 & 0,20 & 0,20 & 0,21 & 0,21 & 0,21 & 0,21 \\
\hline \multirow{2}{*}{ Denmark } & SME & $-0,01$ & $-0,01$ & $-0,01$ & $-0,01$ & $-0,01$ & $-0,01$ & $-0,01$ & $-0,01$ & $-0,01$ & $-0,01$ & $-0,01$ \\
\hline & LF & $-0,01$ & $-0,01$ & $-0,01$ & $-0,01$ & $-0,01$ & $-0,01$ & $-0,01$ & $-0,01$ & $-0,01$ & $-0,01$ & $-0,01$ \\
\hline \multirow{2}{*}{ France } & SME & 0,21 & 0,43 & 0,43 & 0,43 & 0,44 & 0,44 & 0,45 & 0,45 & 0,45 & 0,43 & 0,43 \\
\hline & LF & 0,21 & 0,43 & 0,43 & 0,43 & 0,44 & 0,44 & 0,45 & 0,45 & 0,45 & 0,43 & 0,43 \\
\hline
\end{tabular}


A., Samoilikova, S., Lieonov, A., Huseynova. Tax Incentives for Innovation in the Context of Macroeconomic Stability: an Analysis of Causality

\begin{tabular}{|c|c|c|c|c|c|c|c|c|c|c|c|c|}
\hline & & & & & & & & & & \multicolumn{3}{|c|}{ Continued Table 1} \\
\hline \multirow{2}{*}{ Hungary } & SME & 0,24 & 0,24 & 0,24 & 0,16 & 0,11 & 0,11 & 0,26 & 0,25 & 0,25 & 0,25 & 0,22 \\
\hline & LF & 0,24 & 0,24 & 0,24 & 0,22 & 0,22 & 0,22 & 0,36 & 0,35 & 0,35 & 0,35 & 0,22 \\
\hline \multirow{2}{*}{ Ireland } & SME & 0,21 & 0,21 & 0,26 & 0,26 & 0,25 & 0,27 & 0,26 & 0,27 & 0,29 & 0,29 & 0,29 \\
\hline & LF & 0,21 & 0,21 & 0,26 & 0,26 & 0,25 & 0,27 & 0,26 & 0,27 & 0,29 & 0,29 & 0,29 \\
\hline \multirow{2}{*}{ Italy } & SME & 0,12 & 0,12 & 0,12 & $-0,02$ & $-0,02$ & $-0,02$ & $-0,02$ & $-0,02$ & 0,04 & 0,04 & 0,09 \\
\hline & LF & 0,12 & 0,12 & 0,12 & $-0,02$ & $-0,02$ & $-0,02$ & $-0,02$ & $-0,02$ & 0,04 & 0,04 & 0,09 \\
\hline \multirow{2}{*}{ Lithuania } & SME & 0,00 & 0,31 & 0,45 & 0,31 & 0,31 & 0,31 & 0,31 & 0,31 & 0,31 & 0,31 & 0,31 \\
\hline & LF & 0,00 & 0,31 & 0,45 & 0,31 & 0,31 & 0,31 & 0,31 & 0,31 & 0,31 & 0,31 & 0,31 \\
\hline The & SME & 0,24 & 0,24 & 0,29 & 0,29 & 0,29 & 0,28 & 0,27 & 0,26 & 0,26 & 0,31 & 0,31 \\
\hline Netherlands & LF & 0,07 & 0,07 & 0,09 & 0,09 & 0,10 & 0,12 & 0,14 & 0,15 & 0,15 & 0,15 & 0,15 \\
\hline \multirow{2}{*}{ Portugal } & SME & 0,28 & 0,28 & 0,41 & 0,41 & 0,41 & 0,41 & 0,41 & 0,40 & 0,39 & 0,39 & 0,39 \\
\hline & LF & 0,28 & 0,28 & 0,41 & 0,41 & 0,41 & 0,41 & 0,41 & 0,40 & 0,39 & 0,39 & 0,39 \\
\hline \multirow{2}{*}{ Slovenia } & SME & 0,04 & 0,04 & 0,04 & 0,08 & 0,08 & 0,20 & 0,19 & 0,19 & 0,19 & 0,19 & 0,21 \\
\hline & LF & 0,04 & 0,04 & 0,04 & 0,08 & 0,08 & 0,20 & 0,19 & 0,19 & 0,19 & 0,19 & 0,21 \\
\hline \multirow{2}{*}{ Spain } & SME & 0,39 & 0,35 & 0,35 & 0,35 & 0,35 & 0,35 & 0,35 & 0,35 & 0,34 & 0,33 & 0,33 \\
\hline & LF & 0,39 & 0,35 & 0,35 & 0,35 & 0,35 & 0,35 & 0,35 & 0,35 & 0,34 & 0,33 & 0,33 \\
\hline The United & SME & 0,11 & 0,18 & 0,18 & 0,18 & 0,23 & 0,28 & 0,28 & 0,28 & 0,29 & 0,29 & 0,27 \\
\hline Kingdom & $\mathrm{LF}$ & 0,10 & 0,11 & 0,11 & 0,11 & 0,09 & 0,09 & 0,09 & 0,09 & 0,10 & 0,10 & 0,10 \\
\hline
\end{tabular}

Note: SME - small and medium enterprises; LF - large firm, subjects of large business

Sources: developed by the authors based on (OECD Science, Technology and R\&D Statistics, 2007-2017).

In some countries, the hidden tax subsidy rates on R\&D costs do not depend on the firm's size (Belgium, the Czech Republic, Denmark, France, Ireland, Italy, Lithuania, Portugal, Slovenia, Spain). In the United Kingdom and the Netherlands, rates for small and medium-sized enterprises are higher than for large enterprises. In contrast, in Hungary, large enterprises' hidden tax subsidy rates are higher than for small and medium-sized enterprises. Under comparing the tax subsidy's hidden rates on R\&D expenditures for small and medium-sized enterprises for 2007-2017, it should be noted that the highest rates since 2008-2009 have been stable in France, Portugal, Spain and Lithuania. In turn, Italy had the lowest negative figures from 2009 to 2014. The situation is similar in Denmark, but with a constant negative rate. At the same time, other countries are characterized by ups and downs. Relatively stable indicators also occur in Belgium, the Czech Republic and France. In 2009, there is a rise in this indicator in most countries. Still, for other periods, a clear general trend is not observed due to the individual characteristics of developing this sample's countries. Accordingly, this study compares the hidden tax subsidy rates on R\&D expenditures for large enterprises for 2007-2017. The highest rates are in France, Portugal and Spain. The lowest - in Italy, Denmark, etc. In most countries, the quality is relatively stable in dynamics, except for Hungary, Slovenia, the Netherlands, Italy and others. There are declines and rises in the change of the studied indicator.

Indicators of R\&D tax incentives also include R\&D tax expenditures and direct government financing of business sector R\&D expenditures (R\&D tax expenditure and direct government funding of BERD (Business enterprise R\&D expenditure)). This set of indicators (Tables 2-3), similarly to the previous one, reflects the level and structure of central government support for the development of R\&D research and direct financing of business sector R\&D expenditures in OECD and eleven non-OECD countries. Estimates of the value of R\&D tax benefits are combined with data on direct funding of business sector $R \& D$ expenditures $(O E C D, R \& D$ tax expenditure and direct government funding of $B E R D)$.

Table 2. Indirect government support through tax breaks on R\&D, \% of GDP

\begin{tabular}{lccccccccccc}
\hline \multicolumn{1}{c}{ Country } & $\mathbf{2 0 0 7}$ & $\mathbf{2 0 0 8}$ & $\mathbf{2 0 0 9}$ & $\mathbf{2 0 1 0}$ & $\mathbf{2 0 1 1}$ & $\mathbf{2 0 1 2}$ & $\mathbf{2 0 1 3}$ & $\mathbf{2 0 1 4}$ & $\mathbf{2 0 1 5}$ & $\mathbf{2 0 1 6}$ & $\mathbf{2 0 1 7}$ \\
\hline Belgium & 0,05 & 0,08 & 0,15 & 0,18 & 0,19 & 0,20 & 0,23 & 0,27 & 0,33 & 0,30 & 0,30 \\
The Czech Republic & 0,03 & 0,03 & 0,03 & 0,03 & 0,05 & 0,05 & 0,06 & 0,05 & 0,06 & 0,05 & 0,05 \\
\hline
\end{tabular}


A., Samoilikova, S., Lieonov, A., Huseynova. Tax Incentives for Innovation in the Context of Macroeconomic Stability: an Analysis of Causality

\section{Continued Table 2}

\begin{tabular}{llllllllllll}
\hline Denmark & 0,00 & 0,00 & 0,00 & 0,00 & 0,00 & 0,01 & 0,01 & 0,02 & 0,02 & 0,02 & 0,02 \\
France & 0,10 & 0,23 & 0,26 & 0,28 & 0,27 & 0,28 & 0,28 & 0,28 & 0,29 & 0,28 & 0,28 \\
Hungary & 0,16 & 0,18 & 0,17 & 0,17 & 0,17 & 0,12 & 0,14 & 0,16 & 0,15 & 0,09 & 0,06 \\
Ireland & 0,08 & 0,08 & 0,13 & 0,13 & 0,15 & 0,16 & 0,23 & 0,28 & 0,27 & 0,25 & 0,15 \\
Italy & 0,02 & 0,03 & 0,03 & 0,00 & 0,00 & 0,01 & 0,00 & 0,00 & 0,05 & 0,08 & 0,16 \\
Lithuania & 0,01 & 0,01 & 0,02 & 0,01 & 0,01 & 0,01 & 0,01 & 0,01 & 0,02 & 0,03 & 0,02 \\
The Netherlands & 0,07 & 0,07 & 0,11 & 0,13 & 0,14 & 0,13 & 0,15 & 0,15 & 0,13 & 0,17 & 0,16 \\
Portugal & 0,08 & 0,08 & 0,10 & 0,09 & 0,10 & 0,09 & 0,09 & 0,10 & 0,11 & 0,11 & 0,12 \\
Slovenia & 0,04 & 0,04 & 0,03 & 0,05 & 0,05 & 0,09 & 0,09 & 0,10 & 0,12 & 0,11 & 0,11 \\
Spain & 0,03 & 0,03 & 0,03 & 0,03 & 0,02 & 0,03 & 0,03 & 0,03 & 0,03 & 0,03 & 0,04 \\
The United Kingdom & 0,05 & 0,06 & 0,07 & 0,07 & 0,07 & 0,08 & 0,09 & 0,16 & 0,20 & 0,22 & 0,21 \\
\hline
\end{tabular}

Sources: developed by the authors based on (OECD Science, Technology and R\&D Statistics, R\&D tax expenditure and direct government funding of BERD, 2007-2017).

Table 3. Direct government funding of BERD, $\%$ of GDP

\begin{tabular}{lccccccccccc}
\hline \multicolumn{1}{c}{ Country } & $\mathbf{2 0 0 7}$ & $\mathbf{2 0 0 8}$ & $\mathbf{2 0 0 9}$ & $\mathbf{2 0 1 0}$ & $\mathbf{2 0 1 1}$ & $\mathbf{2 0 1 2}$ & $\mathbf{2 0 1 3}$ & $\mathbf{2 0 1 4}$ & $\mathbf{2 0 1 5}$ & $\mathbf{2 0 1 6}$ & $\mathbf{2 0 1 7}$ \\
\hline Belgium & 0,07 & 0,08 & 0,09 & 0,11 & 0,09 & 0,09 & 0,08 & 0,09 & 0,09 & 0,08 & 0,06 \\
The Czech Republic & 0,11 & 0,10 & 0,11 & 0,11 & 0,12 & 0,12 & 0,11 & 0,10 & 0,08 & 0,06 & 0,08 \\
Denmark & 0,04 & 0,05 & 0,06 & 0,06 & 0,05 & 0,05 & 0,03 & 0,04 & 0,05 & 0,05 & 0,04 \\
France & 0,12 & 0,15 & 0,12 & 0,12 & 0,11 & 0,11 & 0,12 & 0,11 & 0,13 & 0,11 & 0,12 \\
Hungary & 0,05 & 0,04 & 0,10 & 0,10 & 0,11 & 0,13 & 0,18 & 0,16 & 0,19 & 0,07 & 0,13 \\
Ireland & 0,04 & 0,05 & 0,05 & 0,05 & 0,06 & 0,07 & 0,07 & 0,06 & 0,04 & 0,04 & 0,04 \\
Italy & 0,04 & 0,04 & 0,04 & 0,04 & 0,05 & 0,05 & 0,05 & 0,04 & 0,04 & 0,03 & 0,03 \\
Lithuania & 0,01 & 0,01 & 0,01 & 0,01 & 0,00 & 0,01 & 0,01 & 0,01 & 0,01 & 0,00 & 0,00 \\
The Netherlands & 0,02 & 0,02 & 0,03 & 0,06 & 0,04 & 0,02 & 0,02 & 0,02 & 0,02 & 0,02 & 0,02 \\
Portugal & 0,02 & 0,02 & 0,04 & 0,03 & 0,03 & 0,05 & 0,06 & 0,05 & 0,03 & 0,02 & 0,03 \\
Slovenia & 0,07 & 0,06 & 0,14 & 0,22 & 0,27 & 0,27 & 0,25 & 0,14 & 0,07 & 0,05 & 0,07 \\
Spain & 0,11 & 0,13 & 0,12 & 0,12 & 0,10 & 0,09 & 0,07 & 0,06 & 0,06 & 0,06 & 0,06 \\
The United Kingdom & 0,07 & 0,07 & 0,08 & 0,09 & 0,10 & 0,08 & 0,09 & 0,10 & 0,09 & 0,09 & 0,09 \\
\hline
\end{tabular}

Sources: developed by the authors based on (OECD Science, Technology and R\&D Statistics, R\&D tax expenditure and direct government funding of BERD, 2007-2017).

The largest indirect government support values through tax breaks on R\&D (in \% of GDP) are in France, Belgium, and the United Kingdom, and the lowest in Denmark, Lithuania and Spain. Hungary, France, and the United Kingdom are in the lead in the direct public financing of business sector R\&D expenditures (in \% of GDP). The lowest values are shown by Lithuania, Italy, the Netherlands, and Portugal.

Thus, because of the analysis of tax incentives for R\&D from 2007 to 2017, significant differences in fiscal policy approaches to ensure innovative development in different countries were revealed. They identify those who hold the highest and lowest positions in the hidden rate of tax subsidies on R\&D expenditures, indirect government support through tax benefits, and direct government funding of business sector R\&D expenditures. Accordingly, European countries' experience in tax incentives for $R \& D$ is relevant for Ukraine, given the growing role of innovation in various economic activity areas and its impact on economic growth and investment attractiveness of the state as a whole.

It is appropriate to examine how the above indicators of R\&D tax incentives affect, in particular the hidden tax subsidy rate on R\&D expenditures for small and medium enterprises and large enterprises, tax benefits on R\&D (indirect government support) and direct state funding of expenditures business sector on R\&D, on:

- $\quad$ the amount of research and development costs funded by the business sector;

- $\quad$ the amount of investment in the corporate sector;

- $\quad$ the share of investment in GDP; 
- $\quad$ on the indicator of net international investment position, etc.

Table 4 provides data on research and development expenditures financed by the business sector (Intramural R\&D expenditure (GERD) by the source of funds: Business enterprise sector).

Table 4. Intramural R\&D expenditure (GERD) funding by business enterprise sector, $\%$ of GERD

\begin{tabular}{llllllllllll}
\hline Country & $\mathbf{2 0 0 7}$ & $\mathbf{2 0 0 8}$ & $\mathbf{2 0 0 9}$ & $\mathbf{2 0 1 0}$ & $\mathbf{2 0 1 1}$ & $\mathbf{2 0 1 2}$ & $\mathbf{2 0 1 3}$ & $\mathbf{2 0 1 4}$ & $\mathbf{2 0 1 5}$ & $\mathbf{2 0 1 6}$ & $\mathbf{2 0 1 7}$ \\
\hline Belgium & 61,4 & 61,0 & 58,7 & 57,6 & 60,2 & 60,6 & 60,6 & 60,0 & 58,6 & 61,0 & 63,5 \\
The Czech Republic & 47,2 & 45,0 & 39,8 & 40,8 & 37,7 & 36,4 & 37,6 & 35,9 & 34,5 & 39,5 & 39,3 \\
Denmark & 61,0 & 61,5 & 62,1 & 61,1 & 61,2 & 59,9 & 59,0 & 59,0 & 59,1 & 59,0 & 58,5 \\
France & 52,3 & 50,8 & 52,3 & 53,5 & 55,0 & 55,3 & 55,1 & 55,7 & 56,0 & 56,0 & 56,1 \\
Hungary & 43,9 & 48,3 & 46,4 & 47,4 & 47,5 & 46,9 & 46,8 & 48,3 & 49,7 & 56,4 & 52,7 \\
Ireland & 49,5 & 48,8 & 52,1 & 52,2 & 48,9 & 50,2 & 52,6 & 52,2 & 48,7 & 49,0 & 52,1 \\
Italy & 42,0 & 45,9 & 44,2 & 44,7 & 45,1 & 44,3 & 45,2 & 47,3 & 50,0 & 52,1 & 53,7 \\
Lithuania & 32,8 & 29,3 & 30,8 & 32,4 & 28,2 & 26,5 & 27,5 & 32,7 & 28,5 & 39,0 & 35,4 \\
The Netherlands & 48,8 & 47,0 & 45,1 & 48,0 & 51,1 & 51,6 & 51,1 & 51,1 & 48,6 & 52,0 & 51,6 \\
Portugal & 47,0 & 48,1 & 43,9 & 43,9 & 44,7 & 46,0 & 42,3 & 41,8 & 42,7 & 44,4 & 46,5 \\
Slovenia & 58,3 & 62,8 & 58,0 & 58,4 & 61,2 & 62,2 & 63,8 & 68,4 & 69,2 & 69,2 & 63,1 \\
Spain & 45,5 & 45,0 & 43,4 & 43,0 & 44,3 & 45,6 & 46,3 & 46,4 & 45,8 & 46,7 & 47,8 \\
The United Kingdom & 46,0 & 45,4 & 44,5 & 44,0 & 45,9 & 45,6 & 46,2 & 48,0 & 49,0 & 51,8 & 53,7 \\
\hline
\end{tabular}

Sources: developed by the authors based on (EU Statistical Office Data, Intramural R\&D expenditure by source of funds, 2007-2017).

Table 5 presents the investment by Corporate sector, \% of GFCF, in selected countries. The GFCF (Gross fixed capital formation), the share of which represents the corporate sector's investment, means gross fixed capital, which in the general meaning is identified with the category of investment.

Table 5. Investment by Corporate sector, \% of GFCF

\begin{tabular}{|c|c|c|c|c|c|c|c|c|c|c|c|}
\hline Country & 2007 & 2008 & 2009 & 2010 & 2011 & 2012 & 2013 & 2014 & 2015 & 2016 & 2017 \\
\hline Belgium & 63,42 & 63,90 & 63,23 & 62,76 & 64,71 & 64,37 & 64,83 & 64,00 & 65,37 & 66,15 & 66,34 \\
\hline The Czech Republic & 64,92 & 63,58 & 58,46 & 59,39 & 64,26 & 66,54 & 67,61 & 65,73 & 63,55 & 67,90 & 67,21 \\
\hline Denmark & 54,90 & 57,06 & 60,40 & 57,39 & 57,40 & 58,17 & 60,60 & 60,34 & 61,94 & 63,32 & 62,56 \\
\hline France & 53,35 & 54,35 & 53,01 & 53,57 & 54,97 & 55,21 & 55,26 & 56,71 & 58,28 & 58,74 & 58,75 \\
\hline Hungary & 61,04 & 64,17 & 62,34 & 62,19 & 67,36 & 65,69 & 65,06 & 62,33 & 56,32 & 66,74 & 62,54 \\
\hline Ireland & 42,69 & 41,93 & 57,53 & 60,81 & 69,41 & 78,56 & 77,29 & 79,37 & 85,59 & 89,17 & 87,97 \\
\hline Italy & 50,10 & 50,20 & 46,82 & 49,29 & 51,11 & 51,62 & 51,93 & 54,33 & 55,40 & 56,51 & 57,72 \\
\hline Lithuania & 68,01 & 64,31 & 55,38 & 51,01 & 56,30 & 59,73 & 60,43 & 61,41 & 59,82 & 62,18 & 63,59 \\
\hline The Netherlands & 50,30 & 48,06 & 48,29 & 51,90 & 54,48 & 55,47 & 57,98 & 54,97 & 61,72 & 54,07 & 54,71 \\
\hline Portugal & 59,65 & 60,43 & 58,4 & 52,21 & 58,32 & 60,77 & 63,88 & 65,45 & 65,05 & 68,63 & 68,56 \\
\hline Slovenia & 62,26 & 62,14 & 58,22 & 55,44 & 59,52 & 59,58 & 60,28 & 55,15 & 55,43 & 61,36 & 62,75 \\
\hline Spain & 51,88 & 52,08 & 47,72 & 49,75 & 55,73 & 63,51 & 69,03 & 70,94 & 69,99 & 73,02 & 72,11 \\
\hline The United Kingdom & 60,91 & 59,51 & 58,17 & 58,26 & 59,40 & 61,57 & 61,82 & 60,75 & 61,47 & 62,09 & 61,32 \\
\hline
\end{tabular}

Sources: developed by the authors based on (OECD Data, Investment by Corporate sector, 20072017).

Table 6 demonstrates data on the share of total investment in GDP in the selected countries. This indicator shows investment for the general economy, government, business, and households as a share of GDP used for gross investment (GFCF, expressed as a percentage of GDP for government, business and households).

Table 6. Total investment share of GDP, $\%$

\begin{tabular}{llllllllllll}
\hline \multicolumn{1}{c}{ Country } & $\mathbf{2 0 0 7}$ & $\mathbf{2 0 0 8}$ & $\mathbf{2 0 0 9}$ & $\mathbf{2 0 1 0}$ & $\mathbf{2 0 1 1}$ & $\mathbf{2 0 1 2}$ & $\mathbf{2 0 1 3}$ & $\mathbf{2 0 1 4}$ & $\mathbf{2 0 1 5}$ & $\mathbf{2 0 1 6}$ & $\mathbf{2 0 1 7}$ \\
\hline Belgium & 23,29 & 24,12 & 22,78 & 22,12 & 23,01 & 22,96 & 22,17 & 22,81 & 22,96 & 23,21 & 23,11 \\
\hline
\end{tabular}


A., Samoilikova, S., Lieonov, A., Huseynova. Tax Incentives for Innovation in the Context of Macroeconomic Stability: an Analysis of Causality

\begin{tabular}{|c|c|c|c|c|c|c|c|c|c|c|c|}
\hline & & & & & & & & & \multicolumn{3}{|c|}{ Continued Table } \\
\hline The Czech Republic & 29,49 & 28,96 & 27,05 & 26,91 & 26,45 & 25,91 & 25,06 & 25,13 & 26,47 & 24,93 & 24,77 \\
\hline Denmark & 23,51 & 22,94 & 20,17 & 18,11 & 18,16 & 18,78 & 19,05 & 19,16 & 19,85 & 21,02 & 21,19 \\
\hline France & 23,18 & 23,60 & 22,07 & 22,11 & 22,42 & 22,46 & 22,04 & 21,82 & 21,50 & 21,82 & 22,48 \\
\hline Hungary & 23,73 & 23,37 & 22,69 & 20,23 & 19,65 & 19,25 & 20,84 & 22,10 & 22,28 & 19,66 & 22,23 \\
\hline Ireland & 28,69 & 24,78 & 21,09 & 17,54 & 16,60 & 19,61 & 18,56 & 20,64 & 24.06 & 35,63 & 31,39 \\
\hline Italy & 21,66 & 21,28 & 20,11 & 20,02 & 19,71 & 18,31 & 17,20 & 16,72 & 16,94 & 17,17 & 17,48 \\
\hline Lithuania & 28,58 & 26,04 & 17,86 & 16,91 & 18,51 & 17,36 & 18,45 & 18,89 & 19,62 & 19,86 & 19,99 \\
\hline The Netherlands & 23,29 & 22,12 & 21,31 & 19,70 & 20,14 & 18,76 & 18,36 & 17,59 & 22,11 & 20,00 & 20,14 \\
\hline Portugal & 23,54 & 22,55 & 23,23 & 20,73 & 21,49 & 22,41 & 23,54 & 23,82 & 23,78 & 25,34 & 24,95 \\
\hline Slovenia & 22,51 & 22,85 & 21,20 & 20,57 & 18,42 & 15,82 & 14,75 & 15,03 & 15,52 & 15,49 & 16,78 \\
\hline Spain & 28,65 & 29,44 & 24,13 & 21,08 & 19,94 & 19,03 & 19,63 & 19,11 & 18,65 & 17,39 & 18,32 \\
\hline The United Kingdom & 29,86 & 27,84 & 23,11 & 21,79 & 20,02 & 18,53 & 17,37 & 17,78 & 18,01 & 17,96 & 18,66 \\
\hline
\end{tabular}

Sources: developed by the authors based on (EU Statistical Office Data, Investment share of GDP by institutional sectors, 2007-2017).

Table 7 gives the indicators of the net international investment position of the studied countries. Notably, the net global investment position is a statistical indicator that at some point in time shows the difference between external financial assets and liabilities of the economy as a percentage of GDP. It could be positive or negative. The net international investment position reflects the country's net financial position (assets fewer liabilities) relative to the rest of the world. It allows analyzing the country's external work's stock-flow, based on Eurostat data on the balance of payments statistics.

Table 7. Net international investment position, $\%$ of GDP

\begin{tabular}{|c|c|c|c|c|c|c|c|c|c|c|c|}
\hline Country & 2007 & 2008 & 2009 & 2010 & 2011 & 2012 & 2013 & 2014 & 2015 & 2016 & 2017 \\
\hline Belgium & 33,70 & 42,90 & 58,20 & 56,30 & 51,40 & 40,80 & 43,20 & 44,80 & 45,20 & 54,50 & 56,80 \\
\hline $\begin{array}{l}\text { The Czech } \\
\text { Republic }\end{array}$ & $-36,40$ & $-38,10$ & $-43,90$ & $-46,00$ & $-45,20$ & $-45,90$ & $-41,40$ & $-36,60$ & $-32,90$ & $-26,90$ & $-25,00$ \\
\hline Denmark & $-5,80$ & $-5,10$ & 0,90 & 12,80 & 27,80 & 36,10 & 37,20 & 43,30 & 33,40 & 52,60 & 55,40 \\
\hline France & $-8,90$ & $-13,80$ & $-14,80$ & $-9,30$ & $-8,70$ & $-12,80$ & $-16,60$ & $-15,60$ & $-12,90$ & $-13,00$ & $-16,60$ \\
\hline Hungary & $-90,60$ & $-100,40$ & $-113,40$ & $-106,70$ & $-104,20$ & $-92,20$ & $-82,20$ & $-80,40$ & $-67,90$ & $-59,60$ & $-55,10$ \\
\hline Ireland & $-31,40$ & $-95,30$ & $-115,70$ & $-113,50$ & $-139,30$ & $-137,80$ & $-133,40$ & $-164,70$ & $-198,40$ & $-171,70$ & $-167,20$ \\
\hline Italy & $-21,10$ & $-21,60$ & $-22,30$ & $-20,10$ & $-18,20$ & $-23,00$ & $-23,30$ & $-21,00$ & $-19,30$ & $-11,90$ & $-7,70$ \\
\hline Lithuania & $-56,40$ & $-52,70$ & $-61,10$ & $-60,00$ & $-53,60$ & $-54,30$ & $-50,60$ & $-46,80$ & $-43,60$ & $-42,90$ & $-37,90$ \\
\hline The Netherlands & $-14,90$ & $-8,00$ & 1,40 & 11,00 & 20,10 & 26,70 & 30,70 & 48,00 & 48,90 & 61,40 & 59,40 \\
\hline Portugal & $-90,10$ & $-97,80$ & $-110,70$ & $-107,20$ & $-104,10$ & $-119,30$ & $-120,20$ & $-123,80$ & $-118,90$ & $-110,50$ & $-110,40$ \\
\hline Slovenia & $-25,50$ & $-39,40$ & $-40,60$ & $-43,10$ & $-39,80$ & $-44,00$ & $-39,30$ & $-38,40$ & $-31,20$ & $-28,90$ & $-24,20$ \\
\hline Spain & $-85,30$ & $-85,40$ & $-97,60$ & $-91,00$ & $-93,80$ & $-88,90$ & $-92,80$ & $-95,90$ & $-88,90$ & $-85,50$ & $-85,50$ \\
\hline $\begin{array}{l}\text { The United } \\
\text { Kingdom }\end{array}$ & $-8,80$ & 8,90 & $-16,20$ & $-7,90$ & $-11,60$ & $-28,10$ & $-17,80$ & $-22,80$ & 22,70 & 0,30 & $-10,00$ \\
\hline
\end{tabular}

Sources: developed by the authors based on (EU Statistical Office Data, Net international investment position, 2007-2017).

Let's determine the strength and nature of the relationship between the indicators of tax incentives for R\&D in different countries, calculating the correlation coefficients, taking into account time lags in 1, 2 and 3 years. Before that, it is necessary to check whether the studied indicators are subject to the normal distribution, using the Shapiro-Wilk test (Table 8).

Table 8. The results of the Shapiro-Wilk test on the subordination of R\&D tax incentives to the law of normal distribution

\begin{tabular}{|c|c|c|c|c|c|c|c|c|c|}
\hline & $\mathbf{W}$ & V & $\mathbf{z}$ & Prob>z & & $\mathbf{W}$ & V & $\mathbf{Z}$ & Prob>z \\
\hline & & Belgium & & & \multicolumn{5}{|c|}{ The Czech Republic } \\
\hline TSR1 & 0.59580 & 6.544 & 4.080 & $0.00002^{*}$ & TSR1 & 0.66609 & 5.406 & 3.579 & $0.00017^{*}$ \\
\hline TSR2 & 0.59580 & 6.544 & 4.080 & $0.00002^{*}$ & TSR2 & 0.66609 & 5.406 & 3.579 & $0.00017^{*}$ \\
\hline
\end{tabular}


A., Samoilikova, S., Lieonov, A., Huseynova. Tax Incentives for Innovation in the Context of Macroeconomic Stability: an Analysis of Causality

\begin{tabular}{|c|c|c|c|c|c|c|c|c|c|}
\hline \multirow[b]{2}{*}{$\begin{array}{l}\mathrm{TI} \\
\text { GF }\end{array}$} & \multirow[b]{2}{*}{$\begin{array}{l}0.95196 \\
0.97954\end{array}$} & \multirow[b]{2}{*}{$\begin{array}{c}0.778 \\
0.331 \\
\text { Denmark }\end{array}$} & \multirow[b]{2}{*}{$\begin{array}{l}-0.437 \\
-1.793\end{array}$} & \multirow[b]{2}{*}{$\begin{array}{l}0.66911 \\
0.96353\end{array}$} & \multirow[b]{2}{*}{$\begin{array}{l}\text { TI } \\
\text { GF }\end{array}$} & \multirow[b]{2}{*}{$\begin{array}{l}0.85877 \\
0.91680\end{array}$} & \multirow[b]{2}{*}{$\begin{array}{l}2.287 \\
1.347 \\
\text { France }\end{array}$} & \multicolumn{2}{|c|}{ Continued Table 8} \\
\hline & & & & & & & & $\begin{array}{l}1.594 \\
0.545\end{array}$ & $\begin{array}{l}0.05551 \\
0.29286\end{array}$ \\
\hline TSR1 & - & - & - & - & TSR1 & 0.31343 & 11.12 & 5.617 & $0.00000^{*}$ \\
\hline TSR2 & & & & & TSR2 & 0.31343 & 11.12 & 5.617 & $0.00000^{*}$ \\
\hline TI & 0.82451 & 2.841 & 2.058 & $0.01977^{\star}$ & $\mathrm{TI}$ & 0.56694 & 7.012 & 4.267 & $0.00001^{*}$ \\
\hline GF & 0.90145 & $\begin{array}{c}1.596 \\
\text { Hungary }\end{array}$ & 0.869 & 0.19251 & GF & 0.89352 & $\begin{array}{l}1.724 \\
\text { Ireland }\end{array}$ & 1.020 & 0.15384 \\
\hline TSR1 & 0.69708 & 4.905 & 3.333 & $0.00043^{*}$ & TSR1 & 0.88961 & 1.787 & 1.092 & 0.13752 \\
\hline TSR2 & 0.74041 & 4.203 & 2.956 & $0.00156^{*}$ & TSR2 & 0.88961 & 1.787 & 1.092 & 0.13752 \\
\hline TI & 0.85219 & 2.393 & 1.689 & $0.04558^{*}$ & $\mathrm{TI}$ & 0.91538 & 1.370 & 0.577 & 0.28200 \\
\hline GF & 0.95256 & $\begin{array}{r}0.768 \\
\text { Italy }\end{array}$ & -0.459 & 0.67675 & GF & 0.77652 & $\begin{array}{c}3.618 \\
\text { Lithuania }\end{array}$ & 3.618 & $0.00462^{\star}$ \\
\hline \multirow{5}{*}{$\begin{array}{l}\text { TSR1 } \\
\text { TSR2 } \\
\text { TI } \\
\text { GF }\end{array}$} & 0.96823 & 0.514 & -1.117 & 0.86810 & TSR1 & 0.93242 & 1.094 & 0.162 & 0.43582 \\
\hline & 0.96823 & 0.514 & -1.117 & 0.86810 & TSR2 & 0.93242 & 1.094 & 0.162 & 0.43582 \\
\hline & 0.73654 & 4.266 & 2.991 & $0.00139^{*}$ & $\mathrm{TI}$ & 0.95126 & 0.789 & -0.413 & 0.66003 \\
\hline & 0.92125 & 1.275 & 0.442 & 0.32922 & GF & 0.87431 & 2.035 & 1.353 & 0.08804 \\
\hline & \multicolumn{3}{|c|}{ The Netherlands } & & & & Portugal & & \\
\hline TSR1 & 0.99319 & 0.110 & -3.305 & 0.99953 & TSR1 & 0.53758 & 7.487 & 4.448 & $0.00000^{*}$ \\
\hline TSR2 & 0.96039 & 0.641 & -0.761 & 0.77656 & TSR2 & 0.53758 & 7.487 & 4.448 & $0.00000^{*}$ \\
\hline $\mathrm{TI}$ & 0.86550 & 2.178 & 1.492 & 0.06784 & $\mathrm{TI}$ & 0.92560 & 1.205 & 0.337 & 0.36809 \\
\hline \multirow[t]{2}{*}{ GF } & \multirow{2}{*}{\multicolumn{2}{|c|}{ Slovenia }} & 3.346 & 0.00041 & GF & 0.89002 & 1.781 & 1.084 & 0.13917 \\
\hline & & & & & & & Spain & & \\
\hline \multirow{5}{*}{$\begin{array}{l}\text { TSR1 } \\
\text { TSR2 } \\
\text { TI } \\
\text { GF }\end{array}$} & 0.75451 & 3.975 & 2.823 & $0.00238^{*}$ & TSR1 & 0.78368 & 3.502 & 2.528 & $0.00574^{*}$ \\
\hline & 0.75451 & 3.975 & 2.823 & $0.00238^{*}$ & TSR2 & 0.78368 & 3.502 & 2.528 & $0.00574^{*}$ \\
\hline & 0.88465 & 1.868 & 1.179 & 0.11913 & $\mathrm{TI}$ & 0.99386 & 0.099 & -3.437 & 0.99971 \\
\hline & 0.84585 & 2.496 & 1.778 & $0.03767^{*}$ & GF & 0.88477 & 1.866 & 1.177 & 0.11956 \\
\hline & \multicolumn{3}{|c|}{ The United Kingdom } & & & & & & \\
\hline & 0.84942 & & 1.729 & $0.04194^{*}$ & & & & & \\
\hline TSR2 & 0.99112 & 0.144 & -2.962 & 0.99847 & & & & & \\
\hline & 0.79815 & 3.268 & 2.370 & $0.00889^{*}$ & & & & & \\
\hline GF & 0.93510 & 1.051 & 0.089 & 0.46469 & & & & & \\
\hline
\end{tabular}

Accordingly, to determine the strength and nature of the relationship between the indicators that obey the normal distribution law (Shapiro-Wilk test result> 0.05), the Pearson correlation coefficient was calculated. Instead, to identify the relationship between indicators that do not obey the normal distribution law (Shapiro-Wilk test result $<0.05$ ), the Spearman correlation coefficient was calculated. Calculations are performed in the STATA software package. Tables 9-12 present the results of the analyses.

Table 9. Identifying the strength and nature of the relationship between R\&D tax incentives and the share of R\&D expenditures financed by the business sector (considering time lags for the period from 2007 to 2017)

\begin{tabular}{|c|c|c|c|c|c|c|c|c|}
\hline \multirow{2}{*}{ Indicator } & \multicolumn{4}{|c|}{ Time lag, years } & \multicolumn{4}{|c|}{ Time lag, years } \\
\hline & 0 & 1 & 2 & 3 & 0 & 1 & 2 & 3 \\
\hline \multicolumn{5}{|c|}{ Belgium } & \multicolumn{4}{|c|}{ The Czech Republic } \\
\hline TSR_SME & 0.3401 & 0.2195 & 0.5510 & 0.8094 & -0.2182 & 0.4364 & 0.9129 & 0.5491 \\
\hline TSR_LF & 0.3401 & 0.2195 & 0.5510 & 0.8094 & -0.2182 & 0.4364 & 0.9129 & 0.5491 \\
\hline $\mathrm{TI}$ & 0.0360 & 0.3666 & 0.7873 & 0.7218 & -0.7833 & -0.6001 & -0.3849 & 0.0095 \\
\hline GF & -0.9215 & -0.1473 & 0.6211 & 0.4052 & 0.0593 & -0.1706 & -0.5281 & -0.7380 \\
\hline
\end{tabular}


A., Samoilikova, S., Lieonov, A., Huseynova. Tax Incentives for Innovation in the Context of Macroeconomic Stability: an Analysis of Causality

\begin{tabular}{|c|c|c|c|c|c|c|c|c|}
\hline & & & & & \multicolumn{4}{|c|}{ Continued Table 9} \\
\hline \multicolumn{5}{|c|}{ Denmark } & \multicolumn{4}{|c|}{ France } \\
\hline TSR_SME & & 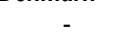 & - & - & -0.0634 & 0.4436 & 0.8872 & 0.9446 \\
\hline TSR_LF & - & - & - & - & -0.0634 & 0.4436 & 0.8872 & 0.9446 \\
\hline $\mathrm{TI}$ & -0.8051 & -0.8051 & -0.6750 & -0.6504 & 0.7545 & 0.7904 & 0.9102 & 0.7545 \\
\hline GF & 0.5567 & 0.1883 & -0.0651 & -0.0717 & -0.7195 & -0.5894 & -0.4917 & -0.4701 \\
\hline \multicolumn{5}{|c|}{ Hungary } & \multicolumn{4}{|c|}{ Ireland } \\
\hline TSR_SME & 0.1473 & 0.7979 & 0.7274 & 0.3805 & 0.2440 & 0.0454 & -0.3148 & -0.0089 \\
\hline TSR_LF & 0.0000 & 0.7509 & 0.7660 & 0.5006 & 0.2440 & 0.0454 & -0.3148 & -0.0089 \\
\hline $\mathrm{TI}$ & -0.3810 & -0.3571 & -0.6429 & -0.5476 & 0.1194 & -0.1420 & -0.3863 & 0.0422 \\
\hline \multirow[t]{2}{*}{ GF } & 0.0464 & 0.4438 & 0.7057 & 0.8522 & 0.5988 & 0.3593 & -0.1317 & -0.6228 \\
\hline & \multicolumn{4}{|c|}{ Italy } & \\
\hline TSR_SME & 0.0543 & -0.1480 & -0.3902 & -0.6994 & -0.1388 & 0.1847 & -0.0752 & Lithuania \\
\hline TSR_LF & 0.0543 & -0.1480 & -0.3902 & -0.6994 & -0.1388 & 0.1847 & -0.0752 & -0.2623 \\
\hline $\mathrm{TI}$ & 0.5476 & 0.4286 & -0.0952 & -0.8095 & 0.5062 & 0.6382 & 0.1600 & -0.1755 \\
\hline GF & -0.6552 & -0.4063 & 0.4440 & 0.6705 & -0.4318 & -0.2649 & -0.2295 & -0.4084 \\
\hline \multicolumn{5}{|c|}{ The Netherlands } & \multicolumn{4}{|c|}{ Portugal } \\
\hline TSR_SME & 0.3547 & 0.6635 & 0.6889 & 0.3282 & -0.0652 & -0.3712 & -0.3546 & -0.4124 \\
\hline TSR_LF & 0.6633 & 0.6485 & 0.5449 & 0.3592 & -0.0652 & -0.3712 & -0.3546 & -0.4124 \\
\hline $\mathrm{TI}$ & 0.6664 & 0.8244 & 0.7824 & 0.4747 & -0.2837 & 0.1580 & 0.3773 & 0.0520 \\
\hline GF & -0.4051 & -0.1964 & -0.2455 & -0.0614 & -0.6472 & -0.8634 & -0.2703 & 0.4140 \\
\hline \multicolumn{5}{|c|}{ Slovenia } & \multicolumn{4}{|c|}{ Spain } \\
\hline TSR_SME & 0.3212 & 0.7451 & 0.8078 & 0.8513 & -0.7011 & -0.7638 & -0.5774 & -0.5774 \\
\hline TSR_LF & 0.3212 & 0.7451 & 0.8078 & 0.8513 & -0.7011 & -0.7638 & -0.5774 & -0.5774 \\
\hline TI & 0.8432 & 0.8265 & 0.7392 & 0.6014 & 0.5 & -0.0293 & -0.3038 & -0.1250 \\
\hline GF & -0.7545 & -0.1198 & 0.5988 & 0.8982 & -0.8017 & -0.9079 & -0.8469 & -0.7010 \\
\hline \multicolumn{5}{|c|}{ The United Kingdom } & & & & \\
\hline TSR_SME & 0.5279 & 0.9266 & 0.9512 & 0.9512 & & & & \\
\hline TSR_LF & -0.1767 & -0.2761 & -0.4809 & -0.7275 & & & & \\
\hline $\mathrm{TI}$ & 0.9524 & 0.9762 & 0.9762 & 0.9762 & & & & \\
\hline GF & 0.2883 & 0.5095 & 0.7211 & 0.7533 & & & & \\
\hline \multicolumn{9}{|c|}{$\begin{array}{l}\text { Notes: TSR_SME - the hidden rate of tax subsidy on R\&D costs for small and medium enterprises; TSR_LF - the hidden rate } \\
\text { of tax subsidy on R\&D costs for large businesses; TI - tax incentives for R\&D (indirect government support); GF - direct } \\
\text { government funding of the business sector's R\&D expenditures. } \\
\text { Sources: developed by the authors. }\end{array}$} \\
\hline \multicolumn{9}{|c|}{$\begin{array}{l}\text { Table 10. The identification of the strength and nature of the relationship between indicators of } \\
\text { tax incentives for R\&D and the amount of investment in the corporate sector (considering time } \\
\text { lags for the period from } 2007 \text { to 2017) }\end{array}$} \\
\hline \multirow{2}{*}{ Indicator } & \multicolumn{4}{|c|}{ Time lag, years } & \multicolumn{4}{|c|}{ Time lag, years } \\
\hline & 0 & 1 & 2 & 3 & 0 & 1 & 2 & 3 \\
\hline \multicolumn{5}{|c|}{ Belgium } & & The Cze & Republic & \\
\hline TSR_SME & 0.6198 & 0.5455 & 0.9129 & 0.8045 & 0.2182 & -0.1091 & -0.1826 & -0.5108 \\
\hline TSR_LF & 0.6198 & 0.5455 & 0.9129 & 0.8045 & 0.2182 & -0.1091 & -0.1826 & -0.5108 \\
\hline $\mathrm{TI}$ & 0.7340 & 0.8156 & 0.9291 & 0.8405 & 0.6963 & 0.8183 & 0.6113 & 0.3584 \\
\hline GF & -0.4995 & 0.2055 & 0.4928 & 0.2902 & -0.3648 & -0.1973 & 0.0478 & -0.0221 \\
\hline & & Denmark & & & & & & \\
\hline TSR_SME & - & - & - & - & -0.0630 & 0.4410 & 0.9449 & 0.9636 \\
\hline TSR_LF & - & - & - & - & -0.0630 & 0.4410 & 0.9449 & 0.9636 \\
\hline TI & 0.9048 & 0.9048 & 0.8383 & 0.8503 & 0.7619 & 0.8333 & 0.8571 & 0.8571 \\
\hline GF & -0.2208 & -0.2131 & -0.4433 & -0.3080 & -0.2819 & -0.4414 & -0.3880 & -0.5546 \\
\hline & & Hungary & & & & & & \\
\hline TSR_SME & -0.3192 & -0.4297 & -0.1818 & 0.5647 & 0.9382 & 0.8777 & 0.8968 & 0.8961 \\
\hline TSR_LF & -0.1565 & -0.5006 & -0.2347 & 0.5006 & 0.9382 & 0.8777 & 0.8968 & 0.8961 \\
\hline TI & -0.1190 & 0.0952 & 0.2857 & 0.4524 & 0.8039 & 0.8852 & 0.9245 & 0.8543 \\
\hline GF & -0.3084 & -0.0833 & -0.2620 & -0.1334 & -0.5952 & -0.2857 & 0.2143 & 0.9048 \\
\hline
\end{tabular}


A., Samoilikova, S., Lieonov, A., Huseynova. Tax Incentives for Innovation in the Context of Macroeconomic Stability: an Analysis of Causality

\begin{tabular}{|c|c|c|c|c|c|c|c|c|}
\hline \multicolumn{9}{|c|}{ Continued Table 10} \\
\hline \multicolumn{5}{|c|}{ Italy } & \multicolumn{4}{|c|}{ Lithuania } \\
\hline TSR_SME & -0.1516 & -0.5107 & -0.6485 & -0.7924 & -0.6235 & -0.6529 & 0.1932 & 0.7474 \\
\hline TSR_LF & -0.1516 & -0.5107 & -0.6485 & -0.7924 & -0.6235 & -0.6529 & 0.1932 & 0.7474 \\
\hline TI & 0.6429 & 0.2857 & -0.2143 & -0.8095 & -0.2384 & 0.0531 & 0.6439 & 0.6801 \\
\hline GF & -0.4630 & -0.1016 & 0.6363 & 0.7642 & -0.5598 & -0.2629 & 0.2770 & 0.1534 \\
\hline \multicolumn{5}{|c|}{ The Netherlands } & \multicolumn{4}{|c|}{ Portugal } \\
\hline TSR SME & 0.1147 & 0.3890 & 0.6042 & 0.5567 & -0.8607 & -0.7835 & -0.0818 & 0.5361 \\
\hline TSR_LF & 0.7873 & 0.7286 & 0.5391 & 0.2183 & -0.8607 & -0.7835 & -0.0818 & 0.5361 \\
\hline $\mathrm{TI}$ & 0.6079 & 0.8004 & 0.7780 & 0.4106 & 0.6599 & 0.5414 & 0.6690 & 0.6349 \\
\hline GF & -0.3571 & -0.2143 & 0.2857 & 0.5714 & 0.0951 & 0.1360 & 0.5854 & 0.7933 \\
\hline \multicolumn{5}{|c|}{ Slovenia } & \multicolumn{4}{|c|}{ Spain } \\
\hline TSR_SME & 0.4980 & 0.2682 & -0.0741 & 0.1730 & -0.7835 & -0.7326 & -0.4124 & -0.5774 \\
\hline TSR_LF & 0.4980 & 0.2682 & -0.0741 & 0.1730 & -0.7835 & -0.7326 & -0.4124 & -0.5774 \\
\hline TI & -0.1607 & 0.1787 & 0.2497 & 0.3099 & 0.3686 & -0.0818 & -0.3511 & -0.3908 \\
\hline GF & -0.1190 & -0.3571 & -0.2619 & -0.2619 & -0.9754 & -0.9588 & -0.8406 & -0.6925 \\
\hline \multicolumn{5}{|c|}{ The United Kingdom } & & & & \\
\hline TSR SME & 0.7979 & 0.6301 & 0.3004 & 0.4506 & & & & \\
\hline TSR_LF & -0.6074 & -0.8017 & -0.5599 & -0.2170 & & & & \\
\hline $\mathrm{Tl}$ & 0.6190 & 0.5238 & 0.5238 & 0.5238 & & & & \\
\hline GF & 0.1764 & 0.6986 & 0.9397 & 0.6741 & & & & \\
\hline
\end{tabular}

Notes: TSR_SME - the hidden rate of tax subsidy on R\&D costs for small and medium enterprises; TSR_LF - the hidden rate of tax subsidy on R\&D costs for large businesses; TI - tax incentives for R\&D (indirect government support); GF - direct government funding of the business sector's R\&D expenditures.

Sources: developed by the authors.

Table 11. Identifying the strength and nature of the relationship between indicators of tax incentives for R\&D and the share of investment in GDP (considering time lags for the period from 2007 to 2017)

\begin{tabular}{|c|c|c|c|c|c|c|c|c|}
\hline \multirow{2}{*}{ Indicator } & \multicolumn{4}{|c|}{ Time lag, years } & \multicolumn{4}{|c|}{ Time lag, years } \\
\hline & 0 & 1 & 2 & 3 & 0 & 1 & 2 & 3 \\
\hline \multicolumn{5}{|c|}{ Belgium } & \multicolumn{4}{|c|}{ The Czech Republic } \\
\hline TSR_SME & 0.2267 & 0.4939 & 0.7806 & 0.6809 & -0.4364 & 0.0000 & 0.0913 & 0.4342 \\
\hline TSR_LF & 0.2267 & 0.4939 & 0.7806 & 0.6809 & -0.4364 & 0.0000 & 0.0913 & 0.4342 \\
\hline $\mathrm{Tl}$ & -0.3080 & -0.1608 & 0.5516 & 0.5541 & -0.8115 & -0.7510 & -0.7039 & -0.5685 \\
\hline GF & -0.5870 & -0.2366 & 0.2858 & -0.1880 & 0.3238 & 0.3095 & 0.0768 & 0.0055 \\
\hline \multicolumn{5}{|c|}{ Denmark } & \multicolumn{4}{|c|}{ France } \\
\hline TSR_SME & - & - & - & - & -0.5070 & -0.8238 & -0.4119 & -0.1989 \\
\hline TSR_LF & - & - & - & - & -0.5070 & -0.8238 & -0.4119 & -0.1989 \\
\hline $\mathrm{TI}$ & 0.9762 & 0.9048 & 0.9102 & 0.9102 & -0.5749 & -0.2156 & 0.1317 & -0.0479 \\
\hline GF & -0.3423 & -0.3898 & -0.3749 & -0.4613 & 0.5384 & 0.0089 & 0.3346 & 0.5079 \\
\hline \multicolumn{5}{|c|}{ Hungary } & \multicolumn{4}{|c|}{ Ireland } \\
\hline TSR_SME & 0.6015 & 0.6138 & 0.3273 & -0.4419 & 0.1706 & 0.4093 & 0.5360 & 0.5905 \\
\hline TSR_LF & 0.3912 & 0.6508 & 0.4447 & -0.4005 & 0.1706 & 0.4093 & 0.5360 & 0.5905 \\
\hline $\mathrm{TI}$ & -0.1429 & -0.6429 & -0.5476 & -0.5238 & 0.0225 & 0.5808 & 0.8793 & 0.9177 \\
\hline GF & -0.2102 & -0.3601 & 0.2302 & 0.3188 & -0.6190 & -0.2619 & 0.2381 & 0.8333 \\
\hline \multicolumn{5}{|c|}{ Italy } & \multicolumn{4}{|c|}{ Lithuania } \\
\hline TSR_SME & 0.5113 & 0.7299 & 0.9208 & 0.9089 & -0.7443 & -0.9335 & 0.2094 & 0.3657 \\
\hline TSR_LF & 0.5113 & 0.7299 & 0.9208 & 0.9089 & -0.7443 & -0.9335 & 0.2094 & 0.3657 \\
\hline TI & 0.0000 & 0.2857 & 0.5238 & 0.6190 & -0.5376 & -0.2508 & 0.6141 & 0.1466 \\
\hline GF & -0.0043 & -0.3321 & -0.8403 & -0.7644 & -0.4088 & -0.1696 & -0.0832 & 0.1221 \\
\hline \multicolumn{5}{|c|}{ The Netherlands } & \multicolumn{4}{|c|}{ Portugal } \\
\hline TSR_SME & -0.3850 & -0.5166 & -0.4923 & -0.3935 & 0.2217 & 0.0412 & -0.5183 & -0.8660 \\
\hline TSR_LF & -0.5653 & -0.2677 & 0.1120 & 0.3248 & 0.2217 & 0.0412 & -0.5183 & -0.8660 \\
\hline $\mathrm{Tl}$ & -0.7194 & -0.4646 & -0.2189 & -0.0832 & -0.5596 & -0.5105 & -0.5006 & -0.6131 \\
\hline GF & 0.2036 & -0.3234 & -0.4551 & -0.6467 & -0.5567 & -0.5839 & -0.6089 & -0.4969 \\
\hline
\end{tabular}


A., Samoilikova, S., Lieonov, A., Huseynova. Tax Incentives for Innovation in the Context of Macroeconomic Stability: an Analysis of Causality

\begin{tabular}{lcccccccc}
\hline \multicolumn{3}{c}{ Slovenia } & \multicolumn{3}{c}{ Continued Table 11 } \\
\hline TSR_SME & -0.7151 & -0.4597 & -0.7042 & -0.7660 & 0.0412 & -0.0468 & -0.2474 & 0.5774 \\
TSR_LF & -0.7151 & -0.4597 & -0.7042 & -0.7660 & 0.0412 & -0.0468 & -0.2474 & 0.5774 \\
TI & -0.8131 & -0.6699 & -0.6840 & -0.7362 & -0.0010 & 0.2670 & 0.4908 & 0.4771 \\
GF & 0.7381 & 0.4048 & -0.0952 & -0.5238 & 0.8086 & 0.6709 & 0.6135 & 0.3510 \\
\multicolumn{3}{c}{ The United Kingdom } & & & & & & \\
TSR_SME & 0.3805 & 0.8401 & 0.9011 & 0.8010 & & & & \\
TSR_LF & 0.3589 & -0.1607 & -0.5983 & -0.9117 & & & & \\
TI & 0.8333 & 0.8571 & 0.8571 & 0.8571 & & & & \\
GF & -0.4961 & 0.0501 & 0.5311 & 0.6643 & & & & \\
\hline
\end{tabular}

Notes: TSR_SME - the hidden rate of tax subsidy on R\&D costs for small and medium enterprises; TSR_LF - the hidden rate of tax subsidy on R\&D costs for large businesses; TI - tax incentives for R\&D (indirect government support); GF - direct government funding of the business sector's R\&D expenditures.

Table 12. Identifying the strength and nature of the relationship between R\&D tax incentives and the net international investment position (considering time lags for the period from 2007 to 2017)

\begin{tabular}{|c|c|c|c|c|c|c|c|c|}
\hline \multirow{2}{*}{ Indicator } & \multicolumn{4}{|c|}{ Time lag, years } & \multicolumn{4}{|c|}{ Time lag, years } \\
\hline & 0 & 1 & 2 & 3 & 0 & 1 & 2 & 3 \\
\hline \multicolumn{5}{|c|}{ Belgium } & \multicolumn{4}{|c|}{ The Czech Republic } \\
\hline TSR_SME & 0.0563 & 0.3273 & 0.2478 & 0.1277 & 0.8729 & 0.4364 & -0.0913 & -0.5874 \\
\hline TSR_LF & 0.0563 & 0.3273 & 0.2478 & 0.1277 & 0.8729 & 0.4364 & -0.0913 & -0.5874 \\
\hline $\mathrm{TI}$ & 0.3879 & 0.1136 & -0.1380 & -0.0034 & 0.3370 & 0.6608 & 0.8889 & 0.9605 \\
\hline GF & 0.1868 & -0.0491 & -0.6331 & -0.5605 & -0.9224 & -0.8586 & -0.5274 & 0.0360 \\
\hline \multicolumn{5}{|c|}{ Denmark } & \multicolumn{4}{|c|}{ France } \\
\hline TSR_SME & - & - & - & - & -0.1901 & -0.2852 & -0.6654 & -0.5779 \\
\hline TSR_LF & - & - & - & - & -0.1901 & -0.2852 & -0.6654 & -0.5779 \\
\hline $\mathrm{TI}$ & 0.7619 & 0.7381 & 0.7066 & 0.7545 & -0.4192 & -0.3353 & -0.5150 & -0.8982 \\
\hline GF & -0.3068 & 0.0122 & 0.2490 & -0.2241 & -0.0715 & 0.0608 & 0.5322 & 0.7477 \\
\hline \multicolumn{5}{|c|}{ Hungary } & \multicolumn{4}{|c|}{ Ireland } \\
\hline TSR_SME & -0.0491 & 0.3314 & 0.4728 & 0.3069 & -0.4619 & -0.2358 & -0.7217 & -0.6990 \\
\hline TSR_LF & -0.0782 & 0.2003 & 0.5189 & 0.3504 & -0.4619 & -0.2358 & -0.7217 & -0.6990 \\
\hline TI & -0.1190 & -0.4048 & -0.3810 & -0.0238 & -0.5573 & -0.5334 & -0.6296 & -0.5057 \\
\hline GF & 0.0676 & 0.0635 & 0.1759 & -0.3038 & 0.7143 & 0.2143 & -0.4048 & -0.9762 \\
\hline \multicolumn{5}{|c|}{ Italy } & \multicolumn{4}{|c|}{ Lithuania } \\
\hline TSR_SME & 0.1814 & 0.0128 & -0.0173 & -0.3572 & 0.0354 & -0.0821 & 0.3885 & 0.4239 \\
\hline TSR_LF & 0.1814 & 0.0128 & -0.0173 & -0.3572 & 0.0354 & -0.0821 & 0.3885 & 0.4239 \\
\hline TI & 0.5952 & 0.5238 & 0.3095 & -0.2857 & 0.5308 & 0.6058 & 0.6864 & 0.3688 \\
\hline GF & -0.8054 & -0.6406 & 0.1266 & 0.2897 & -0.6005 & -0.1842 & 0.0848 & 0.1078 \\
\hline \multicolumn{5}{|c|}{ The Netherlands } & \multicolumn{4}{|c|}{ Portugal } \\
\hline TSR_SME & 0.5578 & 0.4045 & 0.2281 & 0.3818 & 0.0522 & -0.2062 & -0.5455 & -0.8660 \\
\hline TSR_LF & 0.9657 & 0.9757 & 0.9767 & 0.9468 & 0.0522 & -0.2062 & -0.5455 & -0.8660 \\
\hline $\mathrm{Tl}$ & 0.8912 & 0.8676 & 0.8441 & 0.8967 & -0.3565 & -0.1697 & 0.0665 & -0.6200 \\
\hline GF & -0.7381 & -0.8571 & -0.7857 & -0.2381 & -0.8027 & -0.7062 & -0.2321 & -0.0795 \\
\hline \multicolumn{5}{|c|}{ Slovenia } & \multicolumn{4}{|c|}{ Spain } \\
\hline TSR_SME & 0.3959 & 0.6385 & 0.7165 & 0.8895 & -0.8348 & -0.7572 & -0.5009 & -0.0835 \\
\hline TSR_LF & 0.3959 & 0.6385 & 0.7165 & 0.8895 & -0.8348 & -0.7572 & -0.5009 & -0.0835 \\
\hline TI & 0.3385 & 0.8247 & 0.8802 & 0.9746 & 0.2976 & 0.2858 & 0.2489 & 0.4774 \\
\hline GF & -0.8095 & -0.6905 & 0.0238 & 0.5714 & -0.1058 & -0.4608 & -0.5875 & -0.7064 \\
\hline \multicolumn{5}{|c|}{ The United Kingdom } & & & & \\
\hline TSR_SME & -0.2332 & 0.2224 & 0.1502 & 0.1001 & & & & \\
\hline TSR_LF & 0.5886 & 0.4829 & 0.0031 & -0.2660 & & & & \\
\hline TI & 0.1905 & 0.1429 & 0.1429 & 0.1429 & & & & \\
\hline GF & -0.4986 & -0.4687 & 0.0803 & 0.0275 & & & & \\
\hline
\end{tabular}
of tax subsidy on R\&D costs for large businesses; TI - tax incentives for R\&D (indirect government support); GF - direct government funding of the business sector's R\&D expenditures.

Sources: developed by the authors. 

Analysis of Causality

Table 13 shows the summary results of correlation analysis and identification of lags that provoked the maximum relationship between the indicators of tax incentives for R\&D and IP of the country and other macroeconomic indicators.

Table 13. Results of correlation analysis of R\&D tax incentives (lag, years/correlation coefficient)

\begin{tabular}{|c|c|c|c|c|c|c|c|c|c|c|c|c|c|c|c|c|}
\hline & \multicolumn{4}{|c|}{ BERD } & \multicolumn{4}{|c|}{ INV corp } & \multicolumn{4}{|c|}{ INV } & \multicolumn{4}{|c|}{ NIIP } \\
\hline & $\mathrm{R} 1$ & R2 & $\mathrm{TI}$ & GF & R1 & R2 & TI & GF & R1 & R2 & $\mathrm{TI}$ & GF & R1 & R2 & $\mathrm{TI}$ & GF \\
\hline & $3 /$ & $3 /$ & 21 & 01 & 21 & 21 & 21 & 01 & 21 & 21 & 21 & 01 & $1 /$ & $1 /$ & 01 & 21 \\
\hline BEL & 0.8 & 0.8 & 0.8 & -0.9 & 0.9 & 0.9 & 0.9 & -0.5 & 0.8 & 0.8 & 0.6 & -0.6 & 0.3 & 0.3 & 0.4 & -0.6 \\
\hline & 21 & 21 & 01 & 31 & $3 /$ & $3 /$ & $1 /$ & $0 /$ & 01 & 01 & $0 /$ & 01 & $0 /$ & $0 /$ & $3 /$ & $0 /$ \\
\hline CZE & 0.9 & 0.9 & -0.8 & -0.7 & -0.5 & -0.5 & 0.8 & -0.4 & -0.4 & -0.4 & -0.8 & 0.3 & 0.9 & 0.9 & 0.96 & -0.9 \\
\hline DNK & - & - & $\begin{array}{c}0 / \\
-0.8\end{array}$ & $\begin{array}{l}01 \\
0.6\end{array}$ & - & - & $\begin{array}{l}0 / \\
0.9\end{array}$ & $\begin{array}{c}2 / \\
-0.4\end{array}$ & - & - & $\begin{array}{c}0 / \\
0.98\end{array}$ & $\begin{array}{c}3 / \\
-0.5\end{array}$ & - & - & $\begin{array}{l}01 \\
0.8\end{array}$ & $\begin{array}{c}0 / \\
-0.3\end{array}$ \\
\hline FRA & $\begin{array}{l}3 / \\
0.9\end{array}$ & $\begin{array}{l}3 / \\
0.9\end{array}$ & $\begin{array}{l}21 \\
0.9\end{array}$ & $\begin{array}{c}0 / \\
-0.7\end{array}$ & $\begin{array}{c}3 / \\
0.96\end{array}$ & $\begin{array}{c}3 / \\
0.96\end{array}$ & $\begin{array}{l}3 / \\
0.9\end{array}$ & $\begin{array}{c}3 / \\
-0.6\end{array}$ & $\begin{array}{c}1 / \\
-0.8\end{array}$ & $\begin{array}{c}1 / \\
-0.8\end{array}$ & $\begin{array}{c}0 / \\
-0.6\end{array}$ & $\begin{array}{l}0 / \\
0.5\end{array}$ & $\begin{array}{c}21 \\
-0.7\end{array}$ & $\begin{array}{c}21 \\
-0.7\end{array}$ & $\begin{array}{c}3 / \\
-0.9\end{array}$ & $\begin{array}{l}3 / \\
0.7\end{array}$ \\
\hline HUN & $\begin{array}{l}1 / \\
0.8\end{array}$ & $\begin{array}{l}21 \\
0.8\end{array}$ & $\begin{array}{c}21 \\
-0.6\end{array}$ & $\begin{array}{l}3 / \\
0.9\end{array}$ & $\begin{array}{l}3 / \\
0.6\end{array}$ & $\begin{array}{l}3 / \\
0.5\end{array}$ & $\begin{array}{l}3 / \\
0.5\end{array}$ & $\begin{array}{c}0 / \\
-0.3\end{array}$ & $\begin{array}{l}01 \\
0.6\end{array}$ & $\begin{array}{l}1 / \\
0.7\end{array}$ & $\begin{array}{c}1 / \\
-0.7\end{array}$ & $\begin{array}{c}1 / \\
-0.4\end{array}$ & $\begin{array}{l}21 \\
0.5\end{array}$ & $\begin{array}{l}21 \\
0.5\end{array}$ & $\begin{array}{c}1 / \\
-0.4\end{array}$ & $\begin{array}{c}3 / \\
-0.3\end{array}$ \\
\hline JPI & 21 & 21 & 21 & 31 & 01 & 01 & $1 /$ & 01 & 31 & 31 & 21 & 01 & 21 & 21 & 21 & $3 /$ \\
\hline IRL & -0.3 & -0.3 & -0.4 & -0.6 & 0.9 & 0.9 & 0.9 & -0.6 & 0.6 & 0.6 & 0.9 & -0.6 & -0.7 & -0.7 & -0.6 & -0.98 \\
\hline ITA & $3 /$ & $3 /$ & $0 /$ & 01 & 31 & 31 & $3 /$ & 31 & 21 & 21 & $3 /$ & 21 & 31 & $3 /$ & 01 & $0 /$ \\
\hline IIA & -0.7 & -0.7 & 0.6 & -0.7 & -0.8 & -0.8 & -0.8 & 0.8 & 0.9 & 0.9 & 0.6 & -0.8 & -0.4 & -0.4 & 0.6 & -0.8 \\
\hline LTU & $\begin{array}{c}3 / \\
-0.3\end{array}$ & $\begin{array}{c}3 / \\
-0.3\end{array}$ & $\begin{array}{l}1 / \\
0.6\end{array}$ & $\begin{array}{c}0 / \\
-0.5\end{array}$ & $\begin{array}{l}3 / \\
0.8\end{array}$ & $\begin{array}{l}3 / \\
0.8\end{array}$ & $\begin{array}{l}3 / \\
0.7\end{array}$ & $\begin{array}{c}1 / \\
-0.6\end{array}$ & $\begin{array}{c}1 / \\
-0.9\end{array}$ & $\begin{array}{c}1 / \\
-0.9\end{array}$ & $\begin{array}{l}21 \\
0.6\end{array}$ & $\begin{array}{c}0 / \\
-0.4\end{array}$ & $\begin{array}{l}21 \\
0.4\end{array}$ & $\begin{array}{l}21 \\
0.4\end{array}$ & $\begin{array}{l}21 \\
0.7\end{array}$ & $\begin{array}{c}0 / \\
-0.6\end{array}$ \\
\hline NLD & $\begin{array}{l}21 \\
0.7\end{array}$ & $\begin{array}{l}01 \\
0.7\end{array}$ & $\begin{array}{l}1 / \\
0.8\end{array}$ & $\begin{array}{c}0 / \\
-0.4\end{array}$ & $\begin{array}{l}21 \\
0.6\end{array}$ & $\begin{array}{l}01 \\
0.8\end{array}$ & $\begin{array}{l}1 / \\
0.8\end{array}$ & $\begin{array}{l}3 / \\
0.6\end{array}$ & $\begin{array}{l}1 / \\
0.5\end{array}$ & $\begin{array}{c}0 / \\
-0.6\end{array}$ & $\begin{array}{c}0 / \\
-0.7\end{array}$ & $\begin{array}{c}3 / \\
-0.6\end{array}$ & $\begin{array}{l}01 \\
0.6\end{array}$ & $\begin{array}{c}1 / \\
0.98\end{array}$ & $\begin{array}{l}01 \\
0.9\end{array}$ & $\begin{array}{c}1 / \\
-0.9\end{array}$ \\
\hline & $1 /$ & $1 /$ & 21 & $1 /$ & $1 /$ & $1 /$ & $0 /$ & 31 & 31 & 31 & 01 & $0 /$ & $3 /$ & $3 /$ & 31 & $0 /$ \\
\hline PRI & -0.4 & -0.4 & 0.4 & -0.9 & -0.9 & -0.9 & 0.7 & 0.8 & -0.9 & -0.9 & -0.6 & -0.6 & -0.9 & -0.9 & -0.6 & -0.8 \\
\hline & $3 /$ & 31 & 01 & $0 /$ & 01 & 01 & $3 /$ & $1 /$ & $3 /$ & 31 & 01 & 01 & $3 /$ & $3 /$ & $3 /$ & 01 \\
\hline & 0.9 & 0.9 & 0.8 & -0.8 & 0.5 & 0.5 & 0.3 & -0.4 & -0.8 & -0.8 & -0.8 & 0.7 & 0.9 & 0.9 & 0.97 & -0.8 \\
\hline & $1 /$ & $1 /$ & $0 /$ & $1 /$ & $1 /$ & $1 /$ & $0 /$ & $0 /$ & 31 & $3 /$ & 21 & $0 /$ & 01 & $0 /$ & $3 /$ & $3 /$ \\
\hline ES & -0.8 & -0.8 & 0.5 & -0.9 & -0.8 & -0.8 & 0.4 & -0.98 & 0.6 & 0.6 & 0.5 & 0.8 & -0.8 & -0.8 & 0.5 & -0.7 \\
\hline & 21 & $3 /$ & $1 /$ & $3 /$ & 01 & $1 /$ & $0 /$ & 21 & 21 & 31 & $1 /$ & $3 /$ & 01 & $0 /$ & $0 /$ & $0 /$ \\
\hline 0 & 0.95 & -0.7 & 0.98 & 0.8 & 0.8 & -0.8 & 0.6 & 0.94 & 0.9 & -0.9 & 0.9 & 0.7 & -0.2 & 0.6 & 0.2 & -0.5 \\
\hline
\end{tabular}

Notes: - - no connection; BERD - share of R\&D expenditures financed by the business sector; INV corp - share of corporate sector investments; INV - share of investment in GDP; NIIP - net international investment position; R1, R2 - hidden tax subsidy rate on R\&D expenditures for small and medium and large enterprises, respectively; $\mathrm{TI}$ - tax benefits for R\&D; GF - direct state financing of business sector expenditures on R\&D; BEL - Belgium, CZE - the Czech Republic, DNK - Denmark, FRA - France, HUN - Hungary, IRL - Ireland, ITA - Italy, LTU - Lithuania, NLD - the Netherlands, PRT- Portugal, SVN - Slovenia, ESP Spain, UK - the United Kingdom.

The Granger test was used to examine the direction of the impact of tax incentives' studied indicators. According to the accepted hypothesis, they affect the indicators of R\&D expenditures financed by the business sector, corporate sector investment, share of investment in GDP and net international investment position (Table 14).

Table 14. Characteristics of the direction of the impact of tax incentives based on the results of the Granger test

\begin{tabular}{|c|c|c|c|c|}
\hline Indicator & $\begin{array}{c}\text { TSR, TI and GF affect } \\
\text { BERD }\end{array}$ & $\begin{array}{c}\text { TSR, TI and GF affect } \\
\text { INV }_{\text {corp }}\end{array}$ & $\begin{array}{l}\text { TSR, TI and GF } \\
\text { affect INV }\end{array}$ & $\begin{array}{c}\text { TSR, TI and GF } \\
\text { affect NIIP }\end{array}$ \\
\hline Belgium & $\begin{array}{c}\mathrm{TSR} \rightarrow \mathrm{BERD} \\
\mathrm{TI} \rightarrow \mathrm{BERD}\end{array}$ & $\begin{array}{c}\mathrm{TSR} \rightarrow \mathrm{INV} \text { corp } \\
\mathrm{TI} \rightarrow \mathrm{INV}_{\text {corp }} \\
\mathrm{GF} \leftarrow \mathrm{INV} \text { corp }\end{array}$ & $\begin{array}{l}\mathrm{TSR} \rightarrow \mathrm{INV} \\
\mathrm{TI} \rightarrow \mathrm{INV}\end{array}$ & $\begin{array}{l}\mathrm{TSR} \leftarrow \mathrm{NIIP} \\
\mathrm{TI} \leftarrow \mathrm{NIIP}\end{array}$ \\
\hline $\begin{array}{l}\text { The Czech } \\
\text { Republic }\end{array}$ & $\begin{array}{l}\mathrm{TSR} \leftrightarrow \mathrm{BERD} \\
\mathrm{GF} \leftarrow \mathrm{BERD}\end{array}$ & $\begin{array}{l}\mathrm{TI} \leftrightarrow \mathrm{INV}_{\text {corp }} \\
\mathrm{GF} \leftarrow \mathrm{INV} \text { corp }\end{array}$ & $\begin{array}{l}\mathrm{TSR} \rightarrow \mathrm{INV} \\
\mathrm{TI} \rightarrow \mathrm{INV} \\
\mathrm{GF} \rightarrow \mathrm{INV}\end{array}$ & $\begin{array}{l}\mathrm{TSR} \rightarrow \mathrm{NIIP} \\
\mathrm{TI} \rightarrow \mathrm{NIIP} \\
\mathrm{GF} \rightarrow \mathrm{NIIP}\end{array}$ \\
\hline
\end{tabular}


A., Samoilikova, S., Lieonov, A., Huseynova. Tax Incentives for Innovation in the Context of Macroeconomic Stability: an Analysis of Causality

\begin{tabular}{|c|c|c|c|c|}
\hline & & & & Continued Table 14 \\
\hline Denmark & - & $\mathrm{TI} \rightarrow \mathrm{INV}$ corp & $\mathrm{TI} \leftrightarrow \mathrm{INV}$ & $\begin{array}{l}\mathrm{TI} \leftarrow \mathrm{NIIP} \\
\mathrm{GF} \leftarrow \mathrm{NIIP}\end{array}$ \\
\hline France & $\mathrm{TI} \rightarrow \mathrm{BERD}$ & $\mathrm{TI} \leftarrow I N V_{\text {corp }}$ & $\begin{array}{c}\mathrm{TSR} \rightarrow \mathrm{INV} \\
\mathrm{TI} \leftrightarrow \mathrm{INV}\end{array}$ & $\begin{array}{l}\mathrm{TI} \leftarrow \mathrm{NIIP} \\
\mathrm{GF} \leftarrow \mathrm{NIIP}\end{array}$ \\
\hline Hungary & $\mathrm{Tl} \leftarrow \mathrm{BERD}$ & $\mathrm{GF} \leftarrow I N V_{\text {corp }}$ & $\begin{array}{l}\text { GF } \rightarrow \text { INV } \\
\text { TSR } \leftarrow \text { INV }\end{array}$ & $\begin{array}{l}\mathrm{TSR} \rightarrow \mathrm{NIIP} \\
\mathrm{TI} \leftarrow \mathrm{NIIP}\end{array}$ \\
\hline Ireland & - & - & $\begin{array}{l}\mathrm{TI} \rightarrow \mathrm{INV} \\
\mathrm{GF} \rightarrow \mathrm{INV}\end{array}$ & $\begin{array}{l}\mathrm{TI} \rightarrow \mathrm{NIIP} \\
\mathrm{GF} \leftarrow \mathrm{NIIP}\end{array}$ \\
\hline Italy & - & $\begin{array}{c}\mathrm{TSR} \leftarrow I N V_{\text {corp }} \\
\mathrm{TI} \leftarrow I N V_{\text {corp }}\end{array}$ & $\begin{array}{c}\mathrm{TSR} \rightarrow \mathrm{INV} \\
\mathrm{Tl} \leftarrow \mathrm{INV} \\
\mathrm{GF} \leftrightarrow \mathrm{INV}\end{array}$ & $\begin{array}{c}\mathrm{TSR} \rightarrow \mathrm{NIIP} \\
\mathrm{TI} \rightarrow \mathrm{NIIP}\end{array}$ \\
\hline Lithuania & $\mathrm{TI} \rightarrow \mathrm{BERD}$ & $\begin{array}{l}\mathrm{TSR} \rightarrow \mathrm{INV}_{\text {corp }} \\
\mathrm{TI} \rightarrow \mathrm{INV} \text { corp }\end{array}$ & $\begin{array}{c}\mathrm{TSR} \leftrightarrow \mathrm{INV} \\
\mathrm{TI} \rightarrow \mathrm{INV}\end{array}$ & $\begin{array}{l}\mathrm{TSR} \rightarrow \mathrm{NIIP} \\
\mathrm{TI} \rightarrow \mathrm{NIIP} \\
\mathrm{GF} \rightarrow \mathrm{NIIP}\end{array}$ \\
\hline The Netherlands & $\begin{array}{c}\mathrm{TSR} \rightarrow \mathrm{BERD} \\
\mathrm{TI} \rightarrow \mathrm{BERD} \\
\mathrm{GF} \leftrightarrow \mathrm{BERD}\end{array}$ & $\begin{array}{c}\mathrm{TSR} \rightarrow \mathrm{INV} \text { corp } \\
\mathrm{TI} \rightarrow \mathrm{INV} \text { corp } \\
\mathrm{GF} \leftarrow \mathrm{INV}_{\text {corp }}\end{array}$ & - & $\begin{array}{c}\text { TSR } \leftrightarrow \mathrm{NIIP} \\
\mathrm{GF} \rightarrow \mathrm{NIIP}\end{array}$ \\
\hline Portugal & $\mathrm{GF} \leftrightarrow \mathrm{BERD}$ & $\mathrm{GF} \leftarrow \mathrm{INV}$ corp & $\mathrm{TSR} \rightarrow \mathrm{INV}$ & $\mathrm{TI} \leftarrow \mathrm{NIIP}$ \\
\hline Slovenia & - & $\begin{array}{c}\text { TSR } \rightarrow \text { INV } \text { corp } \\
\mathrm{TI} \rightarrow \mathrm{INV}_{\text {corp }}\end{array}$ & GF «INV & $\begin{array}{l}\mathrm{TI} \rightarrow \mathrm{NIIP} \\
\mathrm{GF} \leftrightarrow \mathrm{NIIP}\end{array}$ \\
\hline Spain & $\begin{array}{l}\mathrm{Tl} \leftrightarrow \mathrm{BERD} \\
\mathrm{GF} \rightarrow \mathrm{BERD}\end{array}$ & $\begin{array}{l}\mathrm{TI} \rightarrow \mathrm{INV} \text { corp } \\
\mathrm{GF} \rightarrow \mathbb{N} V_{\text {corp }}\end{array}$ & $\begin{array}{c}\text { TSR } \leftrightarrow \text { INV } \\
\text { TI } \leftrightarrow \text { INV } \\
\text { GF } \leftarrow \text { INV }\end{array}$ & $\begin{array}{l}\mathrm{TI} \leftarrow \mathrm{NIIP} \\
\mathrm{GF} \rightarrow \mathrm{NIIP}\end{array}$ \\
\hline $\begin{array}{l}\text { The United } \\
\text { Kingdom }\end{array}$ & - & $\mathrm{Tl} \leftarrow I N V_{\text {corp }}$ & $\begin{array}{c}\mathrm{TSR} \rightarrow \mathrm{INV} \\
\mathrm{TI} \leftarrow \mathrm{INV}\end{array}$ & $\begin{array}{c}\mathrm{TSR} \rightarrow \mathrm{NIIP} \\
\mathrm{TI} \rightarrow \mathrm{NIIP} \\
\mathrm{GF} \rightarrow \mathrm{NIIP}\end{array}$ \\
\hline
\end{tabular}

Notes: BERD - the share of R\&D expenditures financed by the business sector; INV corp - the share of corporate sector investments; INV - the share of investment in GDP; NIIP - net international investment position; TSR - the hidden rate of tax subsidy on R\&D expenses; TI - tax incentives for R\&D (indirect government support); GF is direct government funding of the business sector's R\&D expenditures.

Sources: developed by the authors.

Considering the results of the Granger test and the level of statistical significance of the calculated Pearson/Spearman correlation coefficients with time lags from 0 to 3 years, there are the following empirically substantiated conclusions:

1) the impact of the hidden rate of tax subsidy on R\&D expenditures on:

- the share of gross domestic expenditure on R\&D financed by the business sector is highest in the Netherlands without a time lag for LF and with a lag of 2 years for SMEs; in the Czech Republic with a lag of 2 years; and Belgium with a lag of 3 years is direct);

- the share of investments made by the corporate sector is the highest in the Netherlands (for LF) without time lag; in Belgium with a lag of 2 years and in Lithuania with a lag of 3 years; medium - in Slovenia without a time lag; and the Netherlands (for SMEs) with a lag of 2 years (the nature of the impact is direct);

- the share of investment in GDP is the highest in France and Lithuania with a lag of 1 year; in Belgium, Italy and the United Kingdom (for SMEs) with a lag of 2 years; in Portugal and the United Kingdom (for LF) with lag at 3 years; medium - in Spain with a lag of 3 years (the nature of the impact is direct in 4 countries and reversed in 4 countries);

- a net international investment position is the highest in the Czech Republic without a time lag; in the Netherlands (for LF) with a lag of 1 year; medium - in the Netherlands (for SMEs) without time lag; in Great Britain (for LF) with lag in 1 year and Hungary with lag in 2 years (the nature of influence is direct);

2) the impact of tax benefits on R\&D (indirect government support) on: 
- the share of gross domestic expenditure on R\&D financed by the business sector is the highest in the Netherlands with a lag of 1 year; in Belgium and France with a lag of 2 years; medium - in Spain without time lag; and in Lithuania with lag in 1 year (the nature of the impact is direct);

- the share of investments made by the corporate sector is the highest in Denmark without time lag; in the Czech Republic and the Netherlands with a lag of 1 year; in Belgium with a lag of 2 years; in Lithuania with a lag of 3 years (the impact is direct);

- the share of investment in GDP is the highest in the Czech Republic and Denmark without a time lag; in Ireland with a lag of 2 years; medium - in France without time lag; in Belgium, Spain and Lithuania with lag in 2 years (the nature of the impact is direct in 5 countries and reversed in 2 countries);

- a net international investment position is significant in Lithuania with a lag of 2 years; in the Czech Republic and Slovenia with a lag of 3 years; medium - in Italy without a time lag and Ireland with a lag of 2 years (the impact is direct at four countries and vice versa in 1 country);

3) the impact of direct public funding of business sector expenditures on R\&D on:

- $\quad$ the share of gross domestic expenditure on R\&D financed by the business sector is as high as possible in Portugal and Spain with a lag of 1 year (the nature of the impact is reversed);

- $\quad$ the share of investments made by the corporate sector is the highest in Spain without time lag (the nature of the impact is reversed);

- $\quad$ the share of investment in GDP is the highest in Italy with a lag of 2 years; medium - in Ireland without a time lag (the nature of the impact is reversed);

- the net international investment position is the highest in the Czech Republic and Slovenia without time lag; in the Netherlands with a time lag of 1 year; in Spain with a time lag of 3 years; the average - in Lithuania and the UK without time lag (nature of the impact is inverted).

A linear regression model for the time series was built to assess the impact of $R \& D$ tax incentives on the share of gross domestic $R \& D$ expenditures financed by the business sector; the share of investments made by the corporate sector; the share of investment in GDP; the indicator of the international investment position on the example of countries where the significant influence of the vast majority of tax incentives on the studied indicators is empirically confirmed.

The following are considered as control factor variables:

- $\quad$ labour force indicator (\% of the total population aged 15-64) (Table 15);

Table 15. The share of labor resources ( $\%$ of the total population aged 15-64)

\begin{tabular}{lccccccccccc}
\hline & $\mathbf{2 0 0 7}$ & $\mathbf{2 0 0 8}$ & $\mathbf{2 0 0 9}$ & $\mathbf{2 0 1 0}$ & $\mathbf{2 0 1 1}$ & $\mathbf{2 0 1 2}$ & $\mathbf{2 0 1 3}$ & $\mathbf{2 0 1 4}$ & $\mathbf{2 0 1 5}$ & $\mathbf{2 0 1 6}$ & $\mathbf{2 0 1 7}$ \\
\cline { 2 - 30 } BEL & 67,21 & 67,20 & 66,94 & 67,65 & 66,62 & 66,79 & 67,44 & 67,72 & 67,68 & 67,73 & 68,06 \\
CZE & 69,92 & 69,69 & 70,04 & 70,12 & 70,45 & 71,48 & 72,80 & 73,53 & 74,13 & 75,19 & 76,11 \\
DNK & 80,23 & 80,80 & 80,28 & 79,32 & 79,04 & 78,35 & 77,89 & 77,94 & 78,38 & 79,94 & 78,84 \\
FRA & 69,66 & 69,79 & 70,13 & 70,17 & 70,04 & 70,58 & 71,01 & 70,93 & 71,18 & 71,38 & 71,54 \\
HUN & 61,52 & 61,14 & 61,16 & 61,82 & 62,29 & 63,49 & 64,33 & 66,60 & 68,37 & 69,95 & 71,09 \\
IRL & 75,25 & 74,60 & 72,93 & 71,63 & 71,23 & 71,24 & 71,97 & 72,08 & 72,28 & 72,85 & 72,91 \\
ITA & 62,37 & 62,84 & 62,19 & 62,02 & 62,15 & 63,57 & 63,38 & 63,95 & 64,07 & 64,98 & 65,49 \\
LTU & 67,93 & 68,43 & 69,63 & 70,33 & 71,51 & 71,99 & 72,55 & 73,75 & 74,09 & 75,55 & 76,10 \\
NLD & 76,77 & 77,85 & 78,10 & 77,90 & 78,05 & 78,90 & 79,28 & 79,00 & 79,66 & 79,73 & 79,79 \\
PRT & 73,97 & 74,03 & 73,43 & 73,56 & 73,55 & 73,38 & 73,05 & 73,30 & 73,60 & 73,97 & 74,91 \\
SVN & 71,41 & 71,85 & 71,70 & 71,76 & 70,77 & 70,89 & 70,79 & 70,99 & 71,64 & 71,54 & 74,24 \\
ESP & 71,77 & 72,75 & 73,16 & 73,63 & 73,95 & 74,29 & 74,29 & 74,22 & 74,38 & 74,38 & 74,16 \\
UK & 75,40 & 75,68 & 75,49 & 75,25 & 75,35 & 75,87 & 76,25 & 76,55 & 76,79 & 77,16 & 77,42 \\
\hline \multicolumn{7}{c}{ Notes: BEL - Belgium, CZE - The Czech Republic, DNK - Denmark, FRA - France, HUN - Hungary, IRL - Ireland, ITA - }
\end{tabular}

Italy, LTU - Lithuania, NLD - The Netherlands, PRT - Portugal, SVN - Slovenia, ESP - Spain, UK - Great Britain.

Sources: developed by the authors based on (World Bank data, Labor force participation rate, 20072017). 
A., Samoilikova, S., Lieonov, A., Huseynova. Tax Incentives for Innovation in the Context of Macroeconomic Stability: an Analysis of Causality

- $\quad$ inflation rate (GDP deflator, \%) (Table 16).

Table 16. Inflation, GDP deflator, \%

\begin{tabular}{lccccccccccc}
\hline & $\mathbf{2 0 0 7}$ & $\mathbf{2 0 0 8}$ & $\mathbf{2 0 0 9}$ & $\mathbf{2 0 1 0}$ & $\mathbf{2 0 1 1}$ & $\mathbf{2 0 1 2}$ & $\mathbf{2 0 1 3}$ & $\mathbf{2 0 1 4}$ & $\mathbf{2 0 1 5}$ & $\mathbf{2 0 1 6}$ & $\mathbf{2 0 1 7}$ \\
\hline BEL & 1,93 & 1,91 & 0,53 & 1,89 & 1,81 & 1,96 & 1,27 & 0,98 & 1,34 & 1,74 & 1,72 \\
CZE & 3,52 & 2,05 & 2,60 & $-1,43$ & 0,02 & 1,46 & 1,43 & 2,48 & 1,17 & 1,27 & 1,44 \\
DNK & 2,43 & 4,13 & 0,53 & 3,22 & 0,64 & 2,38 & 0,89 & 1,03 & 0,43 & 0,25 & 1,13 \\
FRA & 2,56 & 2,37 & 0,07 & 1,07 & 0,95 & 1,16 & 0,78 & 0,58 & 1,14 & 0,52 & 0,46 \\
HUN & 5,44 & 4,79 & 4,19 & 2,38 & 2,18 & 3,20 & 2,98 & 3,59 & 2,46 & 0,97 & 3,70 \\
IRL & 1,21 & $-0,32$ & $-4,56$ & $-3,15$ & 1,50 & 2,28 & 1,23 & $-0,11$ & 7,79 & $-0,30$ & 1,13 \\
ITA & 2,48 & 2,40 & 1,68 & 0,44 & 1,61 & 1,55 & 1,15 & 0,91 & 0,93 & 1,14 & 0,69 \\
LTU & 8,57 & 9,70 & $-3,30$ & 2,27 & 5,38 & 2,78 & 1,35 & 0,92 & 0,09 & 1,61 & 4,25 \\
NLD & 2,07 & 2,31 & 0,22 & 0,94 & 0,19 & 1,45 & 1,28 & 0,25 & 0,77 & 0,45 & 1,26 \\
PRT & 2,97 & 1,74 & 1,10 & 0,64 & $-0,27$ & $-0,39$ & 2,25 & 0,70 & 2,02 & 1,72 & 1,51 \\
SVN & 4,18 & 4,47 & 3,40 & $-1,03$ & 1,04 & 0,48 & 1,60 & 0,46 & 1,01 & 0,75 & 1,58 \\
ESP & 3,42 & 2,25 & 0,14 & 0,15 & $-0,02$ & $-0,11$ & 0,40 & $-0,22$ & 0,55 & 0,32 & 1,38 \\
UK & 2,58 & 2,88 & 1,65 & 1,53 & 2,04 & 1,66 & 1,90 & 1,83 & 0,58 & 2,14 & 1,89 \\
\hline \multicolumn{7}{c}{ Notes: BEL Belgium, CZE - The Czech Republic, DNK - Denmark, FRA - France, HUN - Hungary, IRL - Ireland, ITA - }
\end{tabular}
Italy, LTU - Lithuania, NLD - The Netherlands, PRT - Portugal, SVN - Slovenia, ESP - Spain, UK -Great Britain.

Sources: developed by the authors based on (World Bank data, Inflation, GDP deflator, 2007-2017).

- $\quad$ the interest rate on long-term liabilities (Maastricht criterion interest rates) (Table 17)

Table 17. Maastricht criterion interest rates, $\%$

\begin{tabular}{lccccccccccc}
\hline & $\mathbf{2 0 0 7}$ & $\mathbf{2 0 0 8}$ & $\mathbf{2 0 0 9}$ & $\mathbf{2 0 1 0}$ & $\mathbf{2 0 1 1}$ & $\mathbf{2 0 1 2}$ & $\mathbf{2 0 1 3}$ & $\mathbf{2 0 1 4}$ & $\mathbf{2 0 1 5}$ & $\mathbf{2 0 1 6}$ & $\mathbf{2 0 1 7}$ \\
\hline BEL & 4,33 & 4,42 & 3,90 & 3,46 & 4,23 & 3,00 & 2,41 & 1,71 & 0,84 & 0,48 & 0,72 \\
CZE & 4,30 & 4,63 & 4,84 & 3,88 & 3,71 & 2,78 & 2,11 & 1,58 & 0,58 & 0,43 & 0,98 \\
DNK & 4,29 & 4,28 & 3,59 & 2,93 & 2,73 & 1,40 & 1,75 & 1,32 & 0,69 & 0,32 & 0,48 \\
FRA & 4,30 & 4,23 & 3,65 & 3,12 & 3,32 & 2,54 & 2,20 & 1,67 & 0,84 & 0,47 & 0,81 \\
HUN & 6,74 & 8,24 & 9,12 & 7,28 & 7,63 & 7,89 & 5,92 & 4,81 & 3,43 & 3,14 & 2,96 \\
IRL & 4,31 & 4,53 & 5,23 & 5,74 & 9,60 & 6,17 & 3,79 & 2,37 & 1,18 & 0,74 & 0,80 \\
ITA & 4,49 & 4,68 & 4,31 & 4,04 & 5,42 & 5,49 & 4,32 & 2,89 & 1,71 & 1,49 & 2,11 \\
LTU & 4,54 & 5,61 & 14,00 & 5,57 & 5,16 & 4,83 & 3,83 & 2,79 & 1,38 & 0,90 & 0,31 \\
NLD & 4,29 & 4,23 & 3,69 & 2,99 & 2,99 & 1,93 & 1,96 & 1,45 & 0,69 & 0,29 & 0,52 \\
PRT & 4,42 & 4,52 & 4,21 & 5,40 & 10,24 & 10,55 & 6,29 & 3,75 & 2,42 & 3,17 & 3,05 \\
SVN & 4,53 & 4,61 & 4,38 & 3,83 & 4,97 & 5,81 & 5,81 & 3,27 & 1,71 & 1,15 & 0,96 \\
ESP & 4,31 & 4,37 & 3,98 & 4,25 & 5,44 & 5,85 & 4,56 & 2,72 & 1,73 & 1,39 & 1,56 \\
UK & 5,06 & 4,50 & 3,36 & 3,36 & 2,87 & 1,74 & 2,03 & 2,14 & 1,79 & 1,22 & 1,18 \\
\hline
\end{tabular}

Notes: BEL - Belgium, CZE - The Czech Republic, DNK - Denmark, FRA - France, HUN - Hungary, IRL - Ireland, ITA Italy, LTU - Lithuania, NLD - The Netherlands, PRT - Portugal, SVN - Slovenia, ESP - Spain, UK -Great Britain.

Sources: developed by the authors based on (EU Statistical Office data, Maastricht criterion interest rates, 2007-2017).

Table 18 shows the results of modelling the impact of R\&D tax incentives on the share of gross domestic R\&D expenditures financed by the business sector on Belgium's example. Notably, modelling provides the generating natural logarithms of variables, given that most indicators of R\&D tax incentives showing the results of the Shapiro-Wilk test (Table 18).

Table 18. The results of building a linear regression model to assess the impact of R\&D tax incentives on the share of gross domestic R\&D expenditure financed by the business sector (on the example of Belgium)

\begin{tabular}{|c|c|c|c|c|c|}
\hline InBERD & Coef. & Robust Std. Err. & $t$ & $P>|t|$ & [95\% Conf. Interval] \\
\hline InTSR & .2447539 & .0510345 & 4.80 & 0.009 & .1030593 .3864485 \\
\hline InGF & -.2618628 & .0292623 & -8.95 & 0.001 & $\begin{array}{lll}-.3431079 & -.1806177 \\
\end{array}$ \\
\hline
\end{tabular}


A., Samoilikova, S., Lieonov, A., Huseynova. Tax Incentives for Innovation in the Context of Macroeconomic Stability: an Analysis of Causality

\begin{tabular}{|c|c|c|c|c|c|c|}
\hline & & & & & \multicolumn{2}{|c|}{ Continued Table 18} \\
\hline L.InL & 1.26965 & .5267353 & 2.41 & $0.074^{*}$ & -.1928018 & 2.732101 \\
\hline L.InIR & .0322829 & .0078011 & 4.14 & 0.014 & .0106236 & .0539422 \\
\hline Inl & .0319198 & .0042003 & 7.60 & 0.002 & .0202578 & .0435817 \\
\hline cons & -1.471753 & 2.173924 & -0.68 & $0.536^{*}$ & -7.507533 & 4.564026 \\
\hline & \multicolumn{6}{|c|}{ Prob $>F=0.0002$, R-squared $=0.9755$} \\
\hline L.InTI & .0235808 & .0083306 & 2.83 & 0.047 & .0004513 & .0467103 \\
\hline InGF & -.2444922 & .0335459 & -7.29 & 0.002 & -.3376304 & -.151354 \\
\hline L.InL & .7908687 & .6812537 & 1.16 & $0.310^{*}$ & -1.100595 & 2.682332 \\
\hline$L \cdot \ln \mid R$ & .0265938 & .0106522 & 2.50 & $0.067^{*}$ & -.0029815 & .0561691 \\
\hline $\operatorname{lnl}$ & .0154909 & .0045189 & 3.43 & 0.027 & .0029444 & .0280374 \\
\hline \multirow[t]{2}{*}{ cons } & .178242 & 2.875912 & 0.06 & $0.954^{*}$ & -7.806569 & 8.163053 \\
\hline & \multicolumn{6}{|c|}{ Prob $>F=0.0007$, R-squared $=0.9638$} \\
\hline
\end{tabular}

Notes: ${ }^{*}$ - the value of the coefficient is not statistically significant $\left.(P>|t|)>0.05\right) ; B E R D-$ the share of R\&D expenditures financed by the business sector; TSR - the hidden rate of tax subsidy on R\&D expenses; TI - tax benefits for R\&D; GF - direct state financing of business sector expenditures on $R \& D ; I$ - inflation rate; IR - the interest rate on long-term liabilities; $L$ - an indicator of labour resources.

Sources: developed by the authors.

The obtained values of Prob> F $=0.0002$, R-squared $=0.9755$ and Prob $>F=0.0007$, R-squared $=$ 0.9638 indicate the adequacy of the constructed models, respectively. Coefficients describing the impact of R\&D tax incentives (InTSR, InGF, InTI) are statistically significant $(P>|t|)<0.05)$. The regression of the effect of the hidden rate of the tax subsidy on R\&D expenditures and direct public funding of business sector R\&D expenditures on the amount of gross domestic R\&D expenditures financed by businesses, for example in Belgium, is as follows:

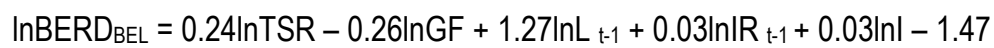

With an increase in the hidden tax subsidy rate on R\&D expenditures by $1 \%$, the value of gross domestic R\&D expenditures financed by businesses would increase by an average of $0.24 \%$. On the other hand, if the volume of direct state financing of business sector expenditures on R\&D rises by $1 \%$, the value of gross domestic spending on R\&D financed by businesses would decrease by $0.26 \%$ on average. The regression of the effect of the tax incentives impact on R\&D and direct public financing of business sector expenditures on R\&D on the amount of gross domestic expenditure of R\&D financed by business, on the example of Belgium, is as follows:

$$
\operatorname{lnBERD} D_{B E L}=\left.0.02 \operatorname{lnT}\right|_{t-1}-0.24 \ln G F+0.79 \operatorname{lnL} t-1+0.03 \ln \left|R_{t-1}+0.02 \ln \right|+0.18
$$

With the growth of R\&D tax benefits by $1 \%$, the gross domestic R\&D expenditures financed by businesses would increase by an average of $0.02 \%$ with a time lag of 1 year. Instead, with an increase in direct government funding for business sector R\&D expenditures by $1 \%$, the gross domestic R\&D expenditures financed by businesses would decrease by an average of $0.24 \%$ (excluding time lag). Table 19 shows the results of modelling the impact of R\&D tax incentives on the gross domestic R\&D expenditures share financed by the business sector (on the Czech Republic example).

Table 19. The results of building a linear regression model to assess the impact of R\&D tax incentives on the share of R\&D gross domestic expenditure financed by the business sector (on the example of the Czech Republic)

\begin{tabular}{lcccccc}
\hline InBERD & Coef. & Robust Std. Err. & $\mathrm{t}$ & $\mathrm{P}>|\mathrm{t}|$ & \multicolumn{2}{c}{$[95 \%$ Conf. Interval] } \\
\hline L.InTSR & 1.973325 & .0993985 & 19.85 & 0.032 & .7103473 & 3.236302 \\
L2.InGF & -.2965119 & .0083393 & -35.56 & 0.018 & -.4024727 & -.1905511 \\
\hline
\end{tabular}


A., Samoilikova, S., Lieonov, A., Huseynova. Tax Incentives for Innovation in the Context of Macroeconomic Stability: an Analysis of Causality

\begin{tabular}{|c|c|c|c|c|c|c|}
\hline & & & & & \multicolumn{2}{|c|}{ Continued Table 19} \\
\hline L1.InL & -7.613918 & .1407899 & -54.08 & 0.012 & -9.402824 & -5.825013 \\
\hline L1.InIR & -.1742106 & .004052 & -42.99 & 0.015 & -.2256959 & -.1227254 \\
\hline L3.Inl & -.0346468 & .001264 & -27.41 & 0.023 & -.0507079 & -.0185857 \\
\hline \multicolumn{7}{|c|}{ Prob $>F=0.0092, R$-squared $=0.9994$} \\
\hline $\ln T$ & -.3447596 & .003299 & -104.50 & 0.000 & -.3589543 & -.330565 \\
\hline L1.InL & -2.93603 & .0652091 & -45.02 & 0.000 & -3.216602 & -2.655458 \\
\hline L1.InIR & -.1266926 & .0016062 & -78.88 & 0.000 & -.1336036 & -.1197815 \\
\hline L3.Inl & -.0073465 & .0001206 & -60.91 & 0.000 & -.0078654 & -.0068275 \\
\hline cons & 15.22055 & .2883256 & 52.79 & 0.000 & 13.97999 & 16.46112 \\
\hline \multicolumn{7}{|c|}{ Prob $>F=0.0000$, R-squared $=0.9999$} \\
\hline
\end{tabular}

Notes: BERD - the share of R\&D expenditures financed by the business sector; TSR - the hidden rate of tax subsidy on R\&D expenses; TI - tax benefits for R\&D; GF - direct state financing of business sector expenditures on R\&D; I - inflation rate; IR - the interest rate on long-term liabilities; $L$ - an indicator of labour resources.

Sources: developed by the authors.

The obtained values of Prob> F $=0.0092, R$-squared $=0.9994$ and Prob $>F=0.0000, R$-squared $=$ 0.9999 indicate the adequacy of the constructed models, respectively. Coefficients describing the impact of R\&D tax incentives (InTSR, InGF, InTI) are statistically significant $(P>|t|)<0.05)$. The regression of the effect of the hidden rate of tax subsidy on R\&D expenditures and direct state financing of business sector R\&D expenditures on the amount of gross domestic R\&D expenditures financed by businesses, as exemplified by the Czech Republic, is as follows:

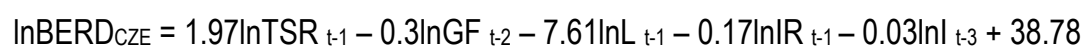

With an increase in the hidden tax subsidy rate on R\&D expenditures by $1 \%$, the value of gross domestic R\&D expenditures financed by businesses would increase by an average of $1.97 \%$ with a time lag of 1 year. Instead, suppose the amount of direct state funding of business sector R\&D expenditures increases by $1 \%$. In that case, the gross domestic R\&D expenditures financed by businesses would decrease by an average of $0.3 \%$ with a time lag of 2 years. The regression equation of the impact of tax incentives on R\&D on the amount of R\&D gross domestic expenditure financed by business (on the Czech Republic example) is as follows:

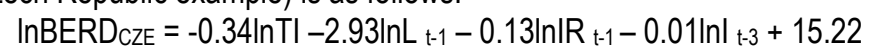

With an increase in R\&D tax benefits by $1 \%$, the gross domestic R\&D expenditures financed by businesses would decrease by an average of $0.34 \%$ (excluding time lag).

Table 20 shows the results of modelling the impact of R\&D tax incentives on the corporate sector's share of investments (on Belgium's example).

Table 20. The results of building a linear regression model to assess the impact of R\&D tax incentives on the share of investment in the corporate sector (on the example of Belgium)

\begin{tabular}{lcccccc}
\hline \multicolumn{1}{c}{$\ln \mid N V_{\text {corp }}$} & Coef. & Robust Std. Err. & $\mathrm{t}$ & $\mathrm{P}>|\mathrm{t}|$ & \multicolumn{2}{c}{$[95 \%$ Conf. Interval] } \\
\hline L3.InTSR & .055544 & .0045841 & 12.12 & 0.001 & .0409555 & .0701326 \\
L.InL & -.1921587 & .2751888 & -0.70 & $0.535^{*}$ & -1.067932 & .683615 \\
L3.InIR & -.030381 & .0045504 & -6.68 & 0.007 & -.0448623 & -.0158996 \\
L2.InI & -.0154491 & .0027643 & -5.59 & 0.011 & -.0242462 & -.006652 \\
cons & 5.129405 & 1.160548 & 4.42 & 0.022 & 1.436022 & 8.822787 \\
& & Prob $>\mathrm{F}=0.0001, \mathrm{R}$-squared & 0.9897 & & \\
L2.InTI & .0375217 & .0018174 & 20.65 & 0.002 & .0297019 & .0453415 \\
InGF & .0373219 & .0028969 & 12.88 & 0.006 & .0248577 & .049786 \\
\hline
\end{tabular}


A., Samoilikova, S., Lieonov, A., Huseynova. Tax Incentives for Innovation in the Context of Macroeconomic Stability: an Analysis of Causality

\begin{tabular}{|c|c|c|c|c|c|}
\hline & & & & & Continued Table 20 \\
\hline L.InL & -.8697899 & .1702914 & -5.11 & 0.036 & $-1.602495 \quad-.1370851$ \\
\hline L3.InIR & -.0301167 & .0010357 & -29.08 & 0.001 & $-.0345729-.0256605$ \\
\hline L2.Inl & -.0182766 & .0007036 & -25.97 & 0.001 & $\begin{array}{lll}-.0213041 & -.0152491\end{array}$ \\
\hline _cons & 8.027993 & .7246892 & 11.08 & 0.008 & $4.909907 \quad 11.14608$ \\
\hline
\end{tabular}

Notes: * the value of the coefficient is not statistically significant $(P>|t|)>0.05)$; INVcorp - the share of corporate sector investments; TSR - the hidden rate of tax subsidy on R\&D expenses; TI - tax benefits for R\&D; GF - direct state financing of business sector expenditures on R\&D; I - inflation rate; IR - the interest rate on long-term liabilities; $L$ - an indicator of labour resources.

Sources: developed by the authors.

The obtained values of Prob> F $=0.0001$, R-squared $=0.9897$ and Prob $>F=0.0001$, R-squared $=$ 0.9986 indicate the adequacy of the constructed models, respectively. Coefficients describing the impact of R\&D tax incentives (InTSR, InGF, $\operatorname{InTI}$ ) are statistically significant $(\mathrm{P}>|\mathrm{t}|)<0.05)$.

The regression equation of the tax subsidy hidden rate impact on R\&D expenditures by the corporate sector's investments share (on Belgium's example), is as follows:

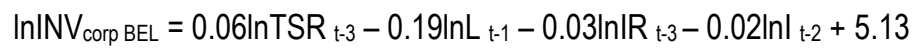

With an increase in the hidden tax subsidy rate on R\&D expenditures by $1 \%$, the corporate sector's investment share would increase by an average of $0.06 \%$ with a time lag of 3 years.

The regression of the impact of tax benefits on R\&D and direct public funding of the business sector's R\&D expenditures by the share of investments made by the corporate sector (on the example of Belgium) is as follows:

$$
\ln \left|N V_{\text {corp BEL }}=0.04 \operatorname{lnTI}\right|_{t-2}+0.04 \ln G F-0.87 \operatorname{lnL} t-1-0.03 \ln \left|R_{t-3}-0.02 \operatorname{ln|}\right|_{t-2}+8.03
$$

With a $1 \%$ increase in R\&D tax benefits, the corporate sector's share of investments would increase by an average of $0.04 \%$ with a time lag of 2 years. Accordingly, if the volume of direct state financing of business sector expenditures on R\&D increases by $1 \%$, the value of gross domestic spending on R\&D financed by the business would increase by an average of $0.04 \%$ (excluding time lag).

Table 21 shows the results of modelling the impact of R\&D tax incentives on the share of the corporate sector's investments (on the Netherlands example).

Table 21. The results of building a linear regression model to assess the impact of R\&D tax incentives on the share of investment in the corporate sector, on the example of the Netherlands (for large businesses)

\begin{tabular}{|c|c|c|c|c|c|}
\hline INV corp & Coef. & Robust Std. Err. & $\mathrm{t}$ & $P>|t|$ & [95\% Conf. Interval] \\
\hline L.InTI & .4036046 & .1303528 & 3.10 & 0.053 & $-.0112363 \quad .8184455$ \\
\hline $\operatorname{lnL}$ & 6.566013 & 1.491651 & 4.40 & 0.022 & 1.81891511 .31311 \\
\hline L.InIR & .0801977 & .0143874 & 5.57 & 0.011 & .0344107 .1259848 \\
\hline L3.Inl & .0320156 & .0063243 & 5.06 & 0.015 & $.0118887 \quad .0521425$ \\
\hline _cons & -23.90539 & 6.676841 & -3.58 & 0.037 & $-45.15408 \quad-2.656699$ \\
\hline \multicolumn{6}{|c|}{ Prob $>F=0.0161$, R-squared $=0.9353$} \\
\hline
\end{tabular}

Notes: INV corp - the share of corporate sector investments; TI - tax benefits for R\&D; I- inflation rate; IR - the interest rate on long-term liabilities; $L$ - an indicator of labour resources.

Sources: developed by the authors.

The obtained values of Prob> $F=0.0161, \mathrm{R}$-squared $=0.9353$ indicate the adequacy of the constructed model. The coefficient describing the impact of the R\&D tax incentives indicator, in particular 
InTI, is statistically significant $(P>|t|)<0.05)$.

The regression equation of the impact of tax incentives on R\&D on the share of investments made by the corporate sector (on the Netherlands example), is as follows:

$$
\operatorname{lnINV} \text { corp NLD }=0.4 \operatorname{lnTI} t_{t-1}+6.57 \operatorname{lnL}+0.08 \ln \mid R_{t-1}+0.03 \operatorname{lnl} t-3-23.9
$$

With a $1 \%$ increase in R\&D tax benefits, the corporate sector's share of investments would increase by an average of $0.4 \%$ with a time lag of 1 year.

Table 22 shows the results of modelling the impact of $R \& D$ tax incentives on the share of investment in GDP on Belgium's example. The obtained values of Prob> F $=0.0050$, R-squared $=0.8852$ indicate the adequacy of the constructed models. The coefficient describing the effect of InTI is statistically significant $(P>|t|)<0.05)$.

The regression equation of the impact of tax incentives on R\&D investment share in GDP (on the example of Belgium) is as follows:

$$
I N V_{B E L}=\left.0.04 \ln T\right|_{t-2}+2.63 \ln L_{t-1}+0.02 \ln \mid R_{t-1}+0.06 \ln -7.91
$$

With the growth of tax benefits on R\&D by $1 \%$, the share of GDP investment would increase by an average of $0.04 \%$ with a time lag of 2 years.

Table 22. The results of building a linear regression model to assess the impact of R\&D tax

\begin{tabular}{|c|c|c|c|c|c|c|}
\hline INV & Coef. & Robust Std. Err. & $t$ & $P>|t|$ & [95\% Con & f. Interval] \\
\hline L2.InTI & .0374955 & .007424 & 5.05 & 0.015 & .0138691 & .0611219 \\
\hline L.InL & 2.630118 & .7482904 & 3.51 & 0.039 & .248724 & 5.011512 \\
\hline L.InIR & .0217146 & .0061964 & 3.50 & 0.039 & .0019948 & .0414345 \\
\hline Inl & .0558579 & .024094 & 2.32 & $0.103^{*}$ & -.0208198 & .1325357 \\
\hline _cons & -7.913952 & 3.154309 & -2.51 & $0.087^{*}$ & -17.95237 & 2.124466 \\
\hline
\end{tabular}
incentives on the share of investment in GDP (on Belgium's example)

Notes: * - the value of the coefficient is not statistically significant (P>|t $\mid)>0.05)$; INV - the share of investment in GDP; TI tax benefits for R\&D; I - inflation rate; IR - the interest rate on long-term liabilities; $L$ - an indicator of labour resources.

Sources: developed by the authors.

Table 23 shows the results of modelling the impact of R\&D tax incentives on the share of investment in GDP on the example of Denmark.

Table 23. The results of building a linear regression model to assess the impact of R\&D tax

\begin{tabular}{|c|c|c|c|c|c|c|}
\hline INV & Coef. & Robust Std. Err. & $t$ & $P>|t|$ & {$[95 \%$ Conf } & f. Interval] \\
\hline L3.InTI & .0357366 & .0058167 & 6.14 & 0.009 & .0172253 & .0542479 \\
\hline L3.InGF & -.2938706 & .0495498 & -5.93 & 0.010 & -.4515602 & -.136181 \\
\hline $\operatorname{lnL}$ & -5.709226 & .8728945 & -6.54 & 0.007 & -8.487166 & -2.931286 \\
\hline $\ln \mid R$ & -.0281018 & .0068519 & -4.10 & 0.026 & -.0499076 & -.0062959 \\
\hline _cons & 27.18846 & 3.669012 & 7.41 & 0.005 & 15.51203 & 38.8649 \\
\hline \multicolumn{7}{|c|}{ Prob $>F=0.0030$, R-squared $=0.9968$} \\
\hline
\end{tabular}
incentives on the share of investment in GDP (on Denmark's example)

Notes: ${ }^{*}$ - the value of the coefficient is not statistically significant $\left.(P>|t|)>0.05\right)$; INV - the share of investment in GDP; TI tax benefits for R\&D; GF - direct state financing of business sector expenditures on R\&D; IR - the interest rate on long-term liabilities; $L$ - an indicator of labour resources.

Sources: developed by the authors.

The obtained values of Prob> F $=0.0030, R$-squared $=0.9968$ indicate the adequacy of the 

Analysis of Causality

constructed model. The coefficients describing the impact of R\&D tax incentives (InGF, InTI) are statistically significant $(P>|t|)<0.05)$.

The regression equation of the impact of tax benefits on R\&D and direct public funding of business sector expenditures on R\&D investment share in GDP (on the example of Denmark) is as follows:

$$
\mathrm{INV}_{\text {DNK }}=0.04 \ln T \mathrm{I}_{\mathrm{t}-3}-0.29 \ln \mathrm{GF}_{\mathrm{t}-3}-5.71 \ln \mathrm{L}-0.03 \ln \operatorname{R}+27.19
$$

With the growth of tax benefits on R\&D by $1 \%$, the share of GDP investment would increase by an average of $0.04 \%$ with a time lag of 3 years. On the other hand, with an immediate increase in the volume of direct state financing of business sector expenditures on R\&D by $1 \%$, the share of GDP investments would decrease by an average of $0.29 \%$ with a time lag of 3 years.

Table 24 shows the results of modelling the impact of R\&D tax incentives on the indicator of the international investment position on the Netherlands' example (for large enterprises).

The obtained values of Prob> F $=0.0001$, R-squared $=0.9948$ indicate the adequacy of the constructed model. The coefficients describing the influence of indicators are statistically significant $(P>\mid$ $\mathrm{t} \mid)<0.05)$.

Table 24. The results of building a linear regression model to assess the impact of R\&D tax incentives on the international investment position on the example of the Netherlands (for large businesses)

\begin{tabular}{|c|c|c|c|c|c|c|}
\hline NIIP & Coef. & Robust Std. Err. & $t$ & $P>|t|$ & \multicolumn{2}{|c|}{ [95\% Conf. Interval] } \\
\hline L2.InTSR & 2.262221 & .1075676 & 21.03 & 0.000 & 1.919893 & 2.604549 \\
\hline $\mathrm{InL}$ & 19.50311 & 4.889334 & 3.99 & 0.028 & 3.943072 & 35.06315 \\
\hline L3.InIR & .6451611 & .0635573 & 10.15 & 0.002 & .4428935 & .8474287 \\
\hline L3.Inl & -.0839681 & .0135131 & -6.21 & 0.008 & -1269727 & -.0409635 \\
\hline _cons & -77.40223 & 21.60525 & -3.58 & 0.037 & -146.1598 & -8.644676 \\
\hline
\end{tabular}

Notes: NIIP - an indicator of the international investment position; TSR - the hidden rate of tax subsidy on R\&D expenses; TI - tax benefits for R\&D; IR - the interest rate on long-term liabilities; $L$ - the indicator of labour resources.

Sources: developed by the authors.

The regression equation for the effect of the hidden tax subsidy rate on R\&D expenditures in the international investment position (on the example of the Netherlands) is as follows:

$\mathrm{NIIPNLD}_{\text {NLD }}=2.26 \ln T \mathrm{SR}_{\mathrm{t}-2}+19.5 \ln L+0.65 \ln \mid \mathrm{R}_{\mathrm{t}-3}-0.08 \ln \mathrm{t}_{\mathrm{t}-3}-77.4$

If the value of the hidden tax subsidy rate on R\&D expenditures increases by $1 \%$, the international investment position will increase by $2.26 \%$ with a time lag of 2 years.

Conclusions. This study based on the application of correlation analysis using the Pearson, Spearman, Shapiro-Wilk and Granger tests. In turn, the Granger test empirically confirmed the impact of high and medium significance of the tax subsidy hidden rate on R\&D expenditures in the gross domestic spending share. R\&D financed by the business sector on the percentage of investments made by the corporate sector on the net international investment position - direct with a time lag of 0-3 years, on the share of investment in GDP - direct in 4 countries and reversed in 4 countries with a lag of 2-3 years; tax benefits for R\&D (indirect government support) - in most countries direct with a lag of 0-3 years; direct state financing of business sector expenditures on R\&D - reversed with a lag of 0-2 years.

The obtained results empirically confirmed the influence of the tax stimulation for innovations on macroeconomic stability indicators. For formalizing the identified causal relationships, the study provides the lag models for those countries where they were most important (Belgium, Denmark, the Netherlands, 
and the Czech Republic).

Author Contributions: methodology, investigation, formal analysis, software, and writing-original draft A. S.; conceptualization, supervision, and editing S. L.; investigation, writing-review, and writingsecond draft A. H.

Funding: The paper was executed in the framework of scientific research work «Quadrocentric recrusive model of Ukrainian unshadow economy to increase its macroeconomic stability» (funding National Research Foundation, Application ID: 2020.02/0238).

\section{References}

Baranovskyi, O. I. (2020). Regulation of functional and structural transformational processes in the financial sector. Financial and credit activity: problems of theory and practice, 1(32), 292-306. [Google Scholar] [CrossRef]

Bloom, N., Griffith, R., \& Van Reenen, J. (2002). Do R\&D tax credits work? Evidence from a panel of countries 19791997. Journal of Public Economics, 85(1), 1-31. [Google Scholar] [CrossRef]

Cappelen, A., Fjærli, E., Foyn, F., Hægeland, T., Møen, J., Raknerud, A., \& Rybalka, M. (2010). Evaluation of the Norwegian R\&D tax credit scheme. Journal of technology management \& innovation, 5(3), 96-109. [GoogleScholar] [CrossRef]

Corchuelo, M. B., \& Martínez-Ros, E. (2010). Who benefits from R\&D tax policy?. Cuadernos de Economía y Dirección de la Empresa, 13(45), 145-170. [Google Scholar] [CrossRef]

Czyżewski, B., Matuszczak, A., \& Miśkiewicz, R. (2019). Public goods versus the farm price-cost squeeze: shaping the sustainability of the EU's common agricultural policy. Technological and Economic Development of Economy, 25(1), 82102. [CrossRef]

Fazio, C., Guzman, J., \& Stern, S. (2020). The impact of state-level research and development tax credits on the quantity and quality of entrepreneurship. Economic Development Quarterly, 34(2), 188-208. [Google Scholar] [CrossRef]

Granger, C. W. (1969). Investigating causal relations by econometric models and cross-spectral methods. Econometrica: journal of the Econometric Society, 424-438. [Google Scholar] [CrossRef]

Guceri, I. (2016). Tax incentives for R\&D. University of Oxford, Centre for Business Taxation. [Google Scholar]

Guceri, I., \& Liu, L. (2015). Effectiveness of fiscal incentives for R\&D: quasi-experimental evidence WP15/12. [Google $\underline{\text { Scholar] }}$

Hall, B., \& Van Reenen, J. (2000). How effective are fiscal incentives for R\&D? A review of the evidence. Research policy, 29(4-5), 449-469. [Google Scholar] [CrossRef]

Hodzic, S., \& Becic, E. (2016). Tax Incentives For R\&D of Large Companies: Empirical Analysis on Croatia. Actual Problems of Economics, 8 (182), 172-179. [GoogleScholar]

Hrytsenko, L. L., Roienko, V., \& Boiarko, I. M. (2018). Institutional background of the role of state in investment processes activation. Financial and credit activities: problems of theory and practice, 1(24), 338-344. [CrossRef]

Inflation, GDP deflator (annual \%). World Bank Data. Retrieved from [Link]

Intramural R\&D expenditure (GERD) by source of funds. EU Statistical Office. Retrieved from [Link]

Investment by Corporate sector, \% of GFCF. OECD Data. Retrieved from [Link]

Investment share of GDP by institutional sectors - \% of GDP. EU Statistical Office. Retrieved from [Link]

James, S. (2013). Tax and non-tax incentives and investments: evidence and policy implications. FIAS, The World Bank Group. [Google Scholar]

Jones, C. I., \& Williams, J. C. (1998). Measuring the social return to R\&D. The Quarterly Journal of Economics, 113(4), 11191135. [Google Scholar] [CrossRef]

Kashintseva, V., Strielkowski, W., Streimikis, J., \& Veynbender, T. (2018). Consumer attitudes towards industrial CO2 capture and storage products and technologies. Energies, 11(10), 2787. [CrossRef]

Klemm, A. (2009). Causes, benefits, and risks of business tax incentives. IMF Working Papers, 1-27. [GoogleScholar]

Koga, T. (2003). Firm size and R\&D tax incentives. Technovation, 23(7), 643-648. [Google Scholar] [CrossRef]

Kuznetsova, A., Pohorelenko, N. (2020). Mechanism of providing financial stability of the banking system of Ukraine. Financial and credit activity-problems of theory and practice, 4. P. 37-47. [CrossRef]

Labor force participation rate, total (\% of total population ages 15-64) (modeled ILO estimate). World Bank Data. Retrieved from [Link]

Lee, C. Y. (2011). The differential effects of public R\&D support on firm R\&D: Theory and evidence from multi-country data. Technovation, 31(5-6), 256-269. [Google Scholar] [CrossRef]

$\mathrm{Li}, \mathrm{W} .$, \& Du, J. (2016). Tax incentives, adjustment costs, and R\&D investment in China. China Journal of Accounting Studies, 4(4), 433-455. [Google Scholar] [CrossRef]

Lopez, L., \& Weber, S. (2017). Testing for Granger causality in panel data. The Stata Journal, 17(4), 972-984. [Google Scholar] [CrossRef] 
Maastricht criterion interest rates. EU Statistical Office. Retrieved from [Link

Miśkiewicz, R, \& Wolniak, R. (2020). Practical Application of the Industry 4.0 Concept in a Steel Company. Sustainability, 12(14), 5776. [CrossRef]

Net international investment position - annual data. EU Statistical Office. Retrieved from [Link]

OECD R\&D Tax Incentive Database. General and country-specific notes. Retrieved from [Link].

Pearson, K. (1896). VII. Mathematical contributions to the theory of evolution.-III. Regression, heredity, and panmixia. Philosophical Transactions of the Royal Society of London. Series A, containing papers of a mathematical or physical character, (187), 253-318. [Google Scholar] [CrossRef]

R\&D tax expenditure and direct government funding of BERD. OECD Science, Technology and R\&D Statistics. Retrieved from [Link]

$\mathrm{R} \& \mathrm{D}$ tax expenditure and direct government funding of BERD. Retrieved from [Link]

Savchenko, T., Basiurkina, N., Rodina, O., \& Kwilinski, A. (2019). Improvement of the assessment methods of product competitiveness of the specialized poultry enterprises. Management Theory and Studies for Rural Business and Infrastructure Development, 41(1), 43-61. [CrossRef]

Shapiro, S. S., \& Wilk, M. B. (1965). An analysis of variance test for normality (complete samples). Biometrika, 52(3/4), 591611. [Google Scholar] [CrossRef]

Simionescu, M., Strielkowski, W. \& Kalyugina, S. (2017). The impact of Brexit on labour migration and labour markets in the United Kingdom and the EU. Terra Economicus, 15(1), 148-156. [CrossRef]

Spearman, C. (1987). The proof and measurement of association between two things. The American journal of psychology, 100(3/4), 441-471. [Google Scholar] [CrossRef]

Strielkowski, W., \& Höschle, F. (2016). Evidence for economic convergence in the EU: The analysis of past EU enlargements. Technological and Economic Development of Economy, 22(4), 617-630. [CrossRef]

Tassey, G. (1996). Choosing government R\&D policies: Tax incentives vs. direct funding. Review of Industrial Organization, 11(5), 579-600. [GoogleScholar] [CrossRef]

Tastan, H. (2015). Testing for spectral Granger causality. The Stata Journal, 15(4), 1157-1166. [GoogleScholar]

Tiutiunyk, I., Kobushko, I., Ivaniy, O., \& Flaumer, A. (2019). Innovations in the Management of Tax Gaps in the Economy: Foreign Economic Component. Marketing and Management of Innovations, 3, 112-125. [GoogleScholar] [CrossRef]

Tkachenko, V., Kwilinski, A., Klymchuk, M., \& Tkachenko, I. (2019). The economic-mathematical development of buildings construction model optimization on the basis of digital economy. Management Systems in Production Engineering, 27(2), 119123. [CrossRef]

Vovchak O. D., Rudevska V. I., Pohorila O. V. (2018). Challenges of inflation targeting in Ukraine: establishing the megaregulator. Financial and credit activities: problems of theory and practice, 1(24), P. 305-311. [CrossRef]

Warda, J. (2001). Measuring the Value of R\&D Tax Treatment in OECD Countries, Special Issue on New Science and Technology Indicators, STI Review No. 27. [GoogleScholar]

Implied tax subsidy rates on R\&D expenditures. OECD Science, Technology and R\&D Statistics. Retrieved from [Link]

Анастасія Самойлікова, к.е.н., Сумський державний університет (Україна);

Сергій Лєонов, д.е.н., профресор, Сумський державний університет (Україна);

Аліда Гусейнова, Ph.D, Азербайджанський Державний Економічний Університет (Азербайджан).

Податкове стимулювання інновацій в контексті макроекономічної стабільності: аналіз причинно-наслідкових зв'язків

У статті розглянуто актуальну проблему податкового стимулювання інновацій, науково-дослідних і дослідноконструкторських робіт, а також його вплив на рівень інноваційного розвитку та макроекономічну стабільність. Дослідження базується на аналізі причинно-наслідкових зв'язків, оціниі сили часових лагів та напрямків взаємного впливу податкових стимулів науково-дослідних і дослідно-конструкторських робіт та макропоказників. Систематизація літературних джерел та підходів до вирішення означеної проблематики вказує на те, що податкові стимули науководослідних і дослідно-конструкторських робіт вивчаються фррагментарно в контексті макроекономічної стабільності. Основною метою дослідження є вдосконалення методологічних основ обгрунтування вибору відповідних інструментів стимулювання інновацій з урахуванням причинно-наслідкових зв'язків податкових стимулів науково-дослідних і дослідноконструкторських робіт та макропоказників. У статті представлені результати динамічного аналізу податкових пільг на науково-дослідні та дослідно-конструкторські роботи. Емпіричне дослідження проведено на основі панельних даних, сформованих для вибірки з 13 європейських країн за 2007-2017 роки. За отриманими результатами визначено значущість, силу та характер взаємозв'язку між досліджуваними показниками та наступними макропоказниками: рівень інноваційного розвитку країни, частка інвестииій у валовому внутрішньому продукті (загалом та в корпоративному секторі, зокрема), чиста міжнародна інвестиційна позиція, частка бізнес-сектору в структурі витрат на науководослідні та дослідно-конструкторські роботи. Коефьіцієнти кореляиії Пірсона та Спірмена було розраховувано залежно від підпорядкування змінних закону нормального розподілу (перевіреного тестом Шапіро-Вілка) на допустимому інтервалі обчислення з урахуванням часових лагів від 0 до 3 років. Причинність досліджуваних показників встановлена за допомогою тесту Грейнджера. Ці розрахунки важливі для розстановки пріоритетів у використанні інструментів 
A., Samoilikova, S., Lieonov, A., Huseynova. Tax Incentives for Innovation in the Context of Macroeconomic Stability: an Analysis of Causality

реалізації інноваційної підтримки. Автори надали найвищий пріоритет встановленню податкових стимулів науководослідних і дослідно-конструкторських робіт, оскільки вплив иього інструменту на всі вивчені макропоказники в більшості країн був прямим, і його ефрект мав місие в найкоротші терміни (з часовим лагом 0-3 роки). Другий nріоритет надано встановленню прихованих ставок субсидій на оподаткування науково-дослідних і дослідноконструкторських робіт бізнесу, оскільки вплив иього показника на більшість досліджуваних показників був статистично значущим та прямим із часовим лагом 0-3 роки. у статті обгрунтовано неефективність прямоі державної фінансової підтримки інновацій, оскільки вплив цього показника на більшість аналізованих макропоказників був зворотним із часовим лагом 0-2 роки. Таким чином, державі доцільніше допомагати підприємиям шляхом надання податкових пільг для забезпечення інноваційного розвитку та макростабільності загалом, ніж шляхом прямого відшкодування витрат. У статті представлено побудовані лагові регресійні моделі для тих країн, де встановлені причинно-наслідкові зв'язки виявилися найбільш статистично значущими (Бельгія, Данія, Нідерланди та Чехія). Вони враховують темпи індрляиї та процентні ставки за довгостроковими зобов'язаннями, а також кількість трудових ресурсів у країні як контрольні змінні.

Ключові слова: інвестиційна позиція, інновації, макроекономічна стабільність, макропоказники, НДДКР, податкові витрати на НДДКР, податкові стимули, податкові субсидії, причинно-наслідкові зв'язки, тест Грейнджера. 